\title{
La Vegetación de los Altos Andes Centrales: Bardas Blancas- Paso Pehuenche (Malargüe, Mendoza, Argentina)
}

\author{
EDUARDO MÉNDEZ1
}

\begin{abstract}
Summary: Vegetation in the high Central Andes: Bardas Blancas-Pehuenche Pass (Malargüe, Mendoza, Argentina). The vegetation occurring between Bardas Blancas and Pehuenche Pass in the south of Mendoza is studied for the purpose of obtaining floristic-ecological information about plant communities. Work is carried out along an altitudinal gradient from 1420 to $2800 \mathrm{~m}$ a.s.l., using phytosociological method. The major plant communities yielded by analyses of comparative survey tables are described. In all, 19 plant communities are revealed, including high- and low-altitude wetlands, and 24 floristic groups characterized by diagnostic, exclusive, preferential and accompanying species. Analysis of dispersal and of hierarchical conglomerates allows confirming the close relationships of plant communities with temperature and altitude. The chorological analysis allowed to identificate two phytogeographic provinces: Patagonia and High Andes. There is a floristic-ecological relationship of phytogeographic environments with life forms. The phytosociological study revealed three vegetation belts: 1- scrubland of Neosparton ephedroides, Larrea nitida, Colliguaja integerrima and Fabiana imbricata, 2- scrubland of Adesmia volckmannii, Chuquiraga oppositifolia, Adesmia pinifolia and Adesmia obovata, and 3- steppe of Azorella monantha, Nassauvia revoluta, Empetrum rubrum and Ochetophila nana. The results may probably allow extrapolation in the north and south of the area.
\end{abstract}

Key words: Argentina, Central Andes, Southern Mendoza, plant communities, phytogeography, phytosociology, vegetation belts.

Resumen: Se estudió la vegetación entre Bardas Blancas y Paso Pehuenche en el Sur Mendocino a fin de obtener información florístico-ecológica de las comunidades vegetales andinas. Se trabajó en un gradiente altitudinal de 1420 a 2800 m s.n.m. y se utilizó el método fitosociológico. Se describen las principales comunidades vegetales que surgen de los análisis de tablas comparativas de relevamientos. Se revela a 19 comunidades vegetales, incluídas las vegas de altura y baja altura, y 24 grupos florísticos caracterizadas por especies diagnósticas, exclusivas, preferenciales y acompañantes. El análisis de dispersión y de conglomerados jerárquicos reafirma las estrechas relaciones de las comunidades vegetales con las temperaturas y altitudes. El análisis corológico identifica a 2 provincias fitogeográficas: Patagonica y Altoandina. Hay una relación florística-ecológica con los ambientes fitogeográficos y las formas de vida. El estudio fitosociológico determina a 3 pisos de vegetación: 1- de los matorrales de Neosparton ephedroides, Larrea nitida, Colliguaja integerrima y Fabiana imbricata, 2- de los matorrales de Adesmia volckmannii, Chuquiraga oppositifolia, Adesmia pinifolia y Adesmia obovata y 3-. de las estepas de Azorella monantha, Nassauvia revoluta, Empetrum rubrum y Ochetophila nana. Los resultados probablemente podrían extrapolarse al $\mathrm{N}$ y $\mathrm{S}$ del área.

Palabras clave: Argentina, Andes Centrales, Sur de Mendoza, comunidades vegetales, fitogeografía, fitosociología, pisos de vegetación.

${ }^{1}$ Botánica y Fitosociología IADIZA CCT CONICET MENDOZA (Ex CRICYT) Avda. Dr. Adrián Ruiz Leal, S/Nº, Parque General San Martín, CC507, CP 5500, Mendoza, Argentina. E-mail: emendez@mendoza-conicet.gob.ar, emendez@ lab.cricyt.edu.ar 


\section{INTRODUCCIÓN}

Si bien no se conocen estudios específicos de la vegetación del área estudiada se tiene información general de otras zonas con relieves geomorfológicos semejantes que ayudan a su interpretación (Hauman, 1918; Ruiz Leal, 1961; Boecher et al., 1963; Cabrera, 1976; Roig, 1976; Ambrosetti et al., 1986; Martínez Carretero \& Méndez, 1992; Martínez Carretero, 2001; Méndez et al., 2006; Méndez, 2004, 2007, 2009, 2010) e incluso los de áreas próximas al sur como las del Manzano (Méndez \& Wuilloud, inédito), del Parque Provincial Tromen (Chiapella \& Ezcurra, 1999) o de Neuquén (Movia et al., 1982).

El área estudiada es de gran interés por ser considerada un paso estratégico binacional, conocido como Corredor Bioceánico (Lacoste, 1998). Esta fue la vía de acceso más empleada por viajeros y exploradores siguiendo el tránsito de los indígenas Pehuenches, Puelches y Araucanos que recorrían el camino, desde y hacia Chile. Cabe señalar que en 1658 ocurre el 1er malón (Pehuenches) sobre la región de Cuyo y fue allí que los aborígenes descubrieron el paso Pehuenche. En 1875 se tiene registros que el valle del río Grande estaba cubierto de pasto abundante y con miles de guanacos. Hacia fines del siglo XVIII el comercio con Chile tomaba cada vez más importancia y el valle se convertía en un lugar estratégico para realizar tráficos de mercadería, ganado y agricultura, y esto lo aseguraba el camino por el paso Pehuenche. Seguramente los nativos fueron los primeros en recolectar las plantas del lugar para usarlas como comestibles, medicinales, y combustibles. Estas colectas de aprovechamiento de la flora y vegetación del área también debieron ocurrir durante las campañas para abrir y mejorar el camino hacia el Paso Pehuenche en el límite con Chile. Todas estas acciones contribuyeron a acrecentar el impacto del área extrayendo los arbustos, y sobre todo las últimas leñas para el fuego. Al respecto no sería extraño que la casi ausencia de Adesmia pinifolia ("leña amarilla") haya sido a causa de esta presión quedando todavía relictos de este matorral, en áreas poco accesibles, como en algunos sectores rocosos de la margen derecha del $\mathrm{A}^{\mathrm{o}}$ Pehuenche, camino al Paso, y actuales testigos de los que debió ser una matorral denso y de incomparable belleza. El área ha sido visitada por botánicos y algunas de sus plantas coleccionadas, destacándose entre ellos Ruiz Leal y Roig cuyas colectas han quedado documentadas con materiales depositados en el Herbario MERL.

Los objetivos de éste trabajo fueron determinar las comunidades vegetales, los pisos de vegetación y las unidades fitogeográficas. Con ello se pretende, una vez más, aportar información a la biodiversidad florística y a la sistemática de las comunidades vegetales. Estos estudios permitirán además ajustar los límites altitudinales de las comunidades vegetales y sus pisos, conocer sus dinamismos, entre otros.

\section{Materiales y Métodos}

\section{El área estudiada}

Se ubica en el Departamento Malargüe y en el Distrito Río Grande (Fig. 1). Se considera en este estudio una transecta representativa a escala 1:50.000 desde Bardas Blancas (1420 m s.n.m.) hasta el sector fronterizo del Paso Pehuenche o Paso del Maule chileno (2525 m s.n.m.) a lo largo de la ruta Nacional $\mathrm{N}^{\circ} 145$ o provincial $\mathrm{N}^{\circ} 224$ bordeando el río Grande y luego sobre el arroyo Pehuenche con una superficie aproximada de 504 $\mathrm{km} 2(12,6 \mathrm{~km}$ de ancho x $40 \mathrm{~km}$ de largo). Esta transecta contiene la gran unidad morfoestructural de montaña en la que podemos diferenciar, por sus desniveles altitudinales, de $\mathrm{E}$ a $\mathrm{W}$ los sectores bajo, intermedio y alto de la montaña (Fig. 1, 2). El Paso Pehuenche une el sur de Mendoza con la VII Región Chilena (Talca, Linares, Curicó, Cauquenes) (Lacoste 1998). Esta transecta además de ofrecer áreas de dominio de uso ganadero y minero, posee un gran valor paisajístico que la hace muy atractiva para el turismo. Próxima al área se encuentra la Ciudad del Pueblo o Perdida, un enclave volcánico del Cerro Trolón, casi oculto y poco conocido por su difícil acceso.

El clima del área se revela con el trazado de las isoyetas e isotermas que permiten diferenciar 3 zonas: 1. Clima BS o Seco de estepa, situado al E, 2. intermedio semiárido y 3. Clima ETH o Polar de tundra al oeste (Norte, 2000) (Fig. 1a) y que además sirven para limitarlas climáticamente, dentro de las unidades agroclimáticas, con los datos termopluviométricos disponibles (De Fina et al., 1964). Al respecto cabe señalar que si 

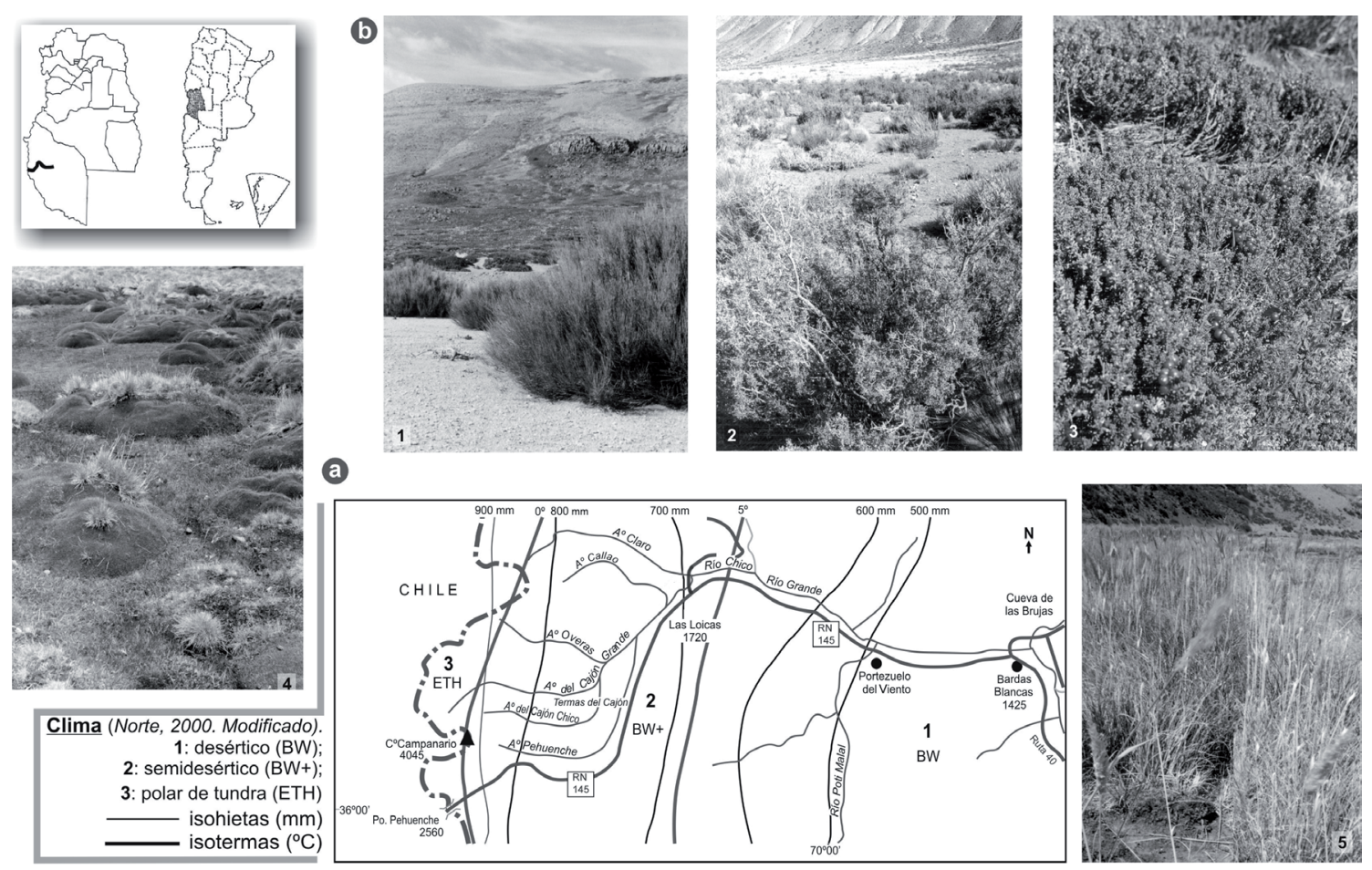

Fig. 1. Localización del área estudiada: a) Cartabón de clima; b) Principales comunidades vegetales. 1- Neosparton ephedroides; 2-Adesmia volckmannii; 3- Empetrum rubrum; 4- Patosia clandestina; 5Cortaderia rudiuscula.

bien el área no posee estaciones meteorológicas cercanas, la temperatura ha sido estudiada con los datos de temperaturas promedios de De Fina et al. (1964) y estimadas, en el gradiente de altitud, por el descenso térmico de $-0,6^{\circ} \mathrm{C}$ por cada $100 \mathrm{~m}$ de elevación (Corte, 1983a, b) (Tabla 3).

La precipitación media anual es superior a 900 $\mathrm{mm}$ en proximidades de la cumbre o del límite con Chile y de unos $300 \mathrm{~mm}$ en la zona del campo principal del río Grande. La rápida disminución de las precipitaciones y nevadas obedece al gradiente topográfico. En la zona montañosa prevalecen las precipitaciones invernales en forma de nieve.

La temperatura media anual de más de $5^{\circ} \mathrm{C}$ está en la parte más baja del área del curso principal del río Grande y la temperatura media anual de $0^{\circ} \mathrm{C}$ en la zona del límite donde la isoterma arrastra el dominio de las nieves eternas o permanentes y el dominio periglaciar manifestadas a niveles menores y en contacto con aquél.

Según la zonificación climática de Minetti \& Corte (1984) el límite inferior geocriogénico se encuentra en nuestras áreas alrededor de los 2600 m s.n.m., lo que coincide con el comienzo de las comunidades altoandinas de Nassauvia y Azorella (Fig. 2). Este piso se caracteriza por tener pendientes de detritos y procesos de criogelifluxión. También se observa permafrost.

La geología del área se conoce bien (Polanski, 1963; Dessanti, 1973). En general se diferencian dos sectores: la del E con un dominio de sedimentos más recientes del Cuaternario o del Terciario y la del W donde dominan materiales de origen Paleozoico, Mesozoico y Cenozoico, entre otros. Hay rocas de edad paleozoica, jurásica, sedimentaria, marina y cretácica. En el valle y terrazas del río Grande hay depósitos eólicos, aluviales y fluviales lacustres del Holoceno (Dessanti, 1973).

Toda el área está representada por la unidad morfoestructural de la Cordillera Principal, y cuyo límite al E, estaría en el río Grande y la Payunia (Capitanelli, 1972; González Díaz \& Fauque, 1993; Bordonaro, 2012). Es posible diferenciar sectores que responden a distintas condiciones de 
Bol. Soc. Argent. Bot. 49 (2) 2014
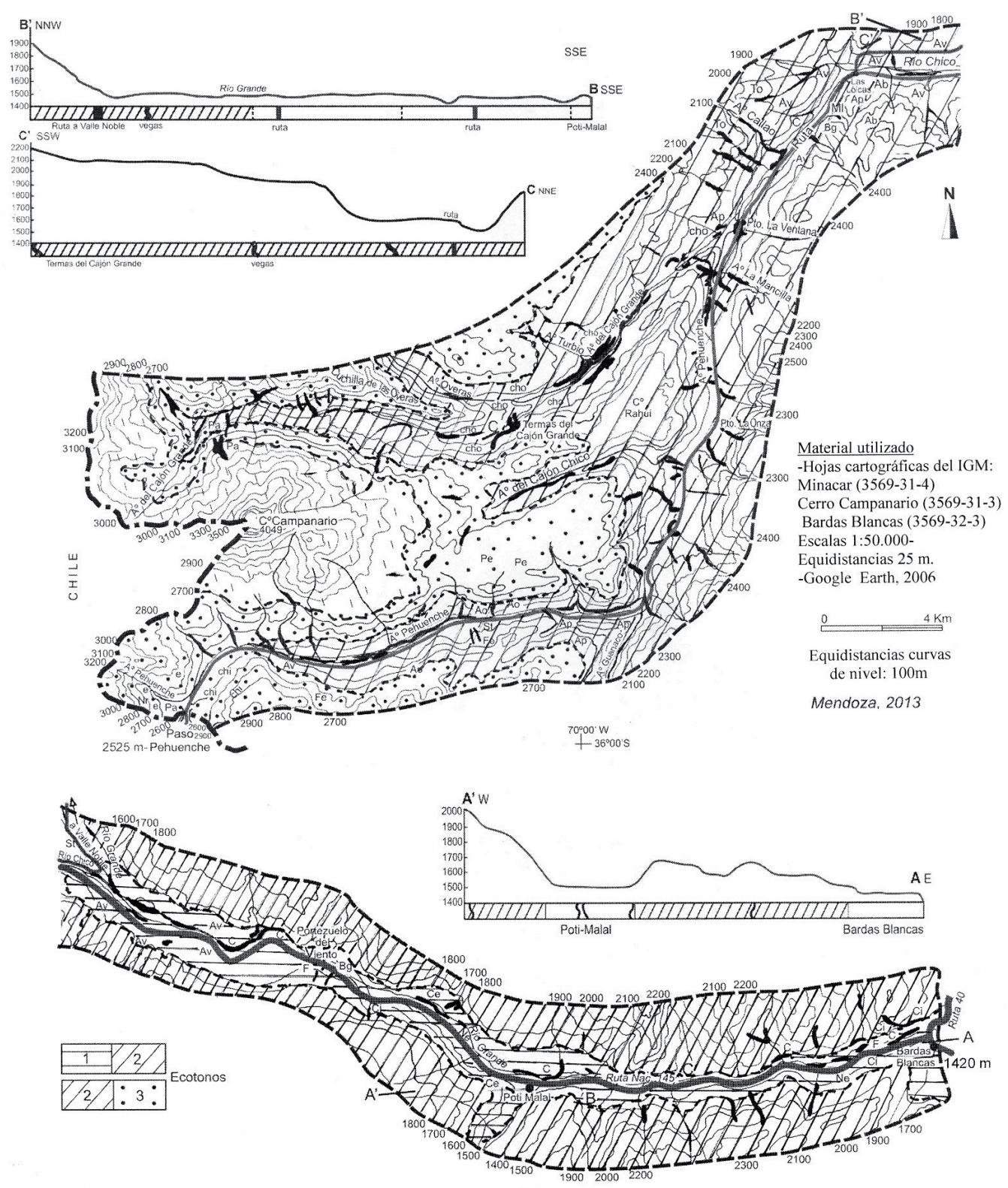

MAPA DE VEGETACIÓN BARDAS BLANCAS- PASO PEHUENCHE (MALARGÜE, MENDOZA, ARGENTINA) Eduardo Méndez IADIZA CCT-Mendoza

REFERENCIAS

Piso de los matorrales de Neosparton ephedroides, Larrea nitida, Colliguaja integerrima y Fabiana imbricata.1420-1600 71 Piso de los matorrales de Adesmia volckmannii, Chuquiraga oppositifolia, Adesmia pinifolia, Adesmia obovata y pastizales $\angle 7$ de Stipa speciosa var. major (=Pappostipa speciosa) y Poa holciformis........................................1500-2600 $\because$. Piso de las estepas de Azorella monantha, Nassauvia revoluta, Empetrum nibrum y Discaria nana....................2500-2800 No visto

OTRAS REFERENCIAS

Ab: Argylia bustillosii. Phil., Ao: Adesmia obovata Clos; Ap: Adesmia pinifolia Gillies ex Hook. \& Arn; Av: Adesmia volcknannii Phil.; Bg: Buddleja globosa Hope; Ce: Centaurea solstitialis L.: C: Cortaderia rudiuscula Stapf.; Chi: Chili otrichum diftussum (G. Fortst.) Kuntze (=C. rosmar ri folium Less.); Cho: Chuquiraga oppositifol ia D.Don; Ci: Colliguaja integerrima Gillies \& Hook; e: Empetrum rubrum Vahl ex Willd.; F. Fabiana imbricata Ruiz \& Pav., Fe: Festuca kurtziana StIves; M1 Malesherbia lira Ga Gay; Ne: Neosparton ephedroides Grise b; Nr. Nassauvia revoluta D. Don; Pa: Patosia clandestina (Phil.) Buchenau; St: Stipa speciosa Trin. \& Rupr var. major (=Pappostipa speciosa); Ta :Tamarix ramosissima Ledeb (- Tamarix gallica auct. Non L.); Pe: Peladero; To: Toscales; V: Vegas.

Fig. 2. Mapa de Vegetación Bardas Blancas-Paso Pehuenche. 


\section{E. Méndez - Vegetación de los altos Andes Centrales}

suelos, clima y pendientes. El sector W más alto del flanco oriental andino de la Cordillera Principal, lo componen relieves profusamente disectados por numerosos cauces con aguas permanentes que desaguan al Arroyo Chico y Pehuenche. El cerro Campanario es el más alto, con 4049 m s.n.m. El sector E mayormente está representado por relieves de menor altura que llega a los 1500 m s.n.m., en las Loicas, y que están fuertemente disectado por una red de cauces temporarios que desaguan al principal del río Grande. En su valle se destacan, entre otras, geoformas mayormente vinculadas a procesos fluviales o eólicos, a planicies de terrazas aluviales, deposiciones o acumulaciones de arenas en taludes y o pendientes. Por otro lado, el área está íntegramente ubicada en la unidad morfoclimática de las grandes montañas dominadas por las masas de aire provenientes del Anticiclón del Pacífico con precipitaciones invernales (Capitanelli, 1972).

Los suelos que dominan al área en general son conglomerados de textura gruesa y permeables. Al este son consolidados compuestos por rocas cristalinas, areniscas y calcáreo y forman delgados mantos donde se desarrolla la vegetación. Suelos originados de rocas cristalinas básicas son localizados al oeste. Existen también texturas muy finas de arcillas y limos. Las arenas son frecuentes en el sector medio del área. Los suelos de las vegas o mallines, son muy húmedos y ricos en materia orgánica, otros en cambio, como los de las partes bajas y de solanas, son cálidos y muy secos. Existen suelos permanentemente congelados (permafrost, Corte, 1983a, b), estructurados, como los de alta montaña o bien con congelamiento estacional y estados intermedios en sectores más bajos. Hay suelos arenosos, secos y profundos en los sitios de médanos más cálidos de los sectores bajos e intermedios, y hasta más fríos en la alta montaña donde hay suelos turbosos y de tundra.

El principal cauce del área es el río Grande con agua permanente. Hay dos grandes redes de drenaje, el de la montaña de gran desarrollo con cursos subparalelos y agua permanente y el menos encauzado y con agua temporarias, en los sectores más bajos. Pequeñas lagunitas de origen endorreico y con agua de los deshielos se sitúan próximas a grandes alturas (2200-2550 m s.n.m.) en el camino al Paso Pehuenche.

Con respecto al uso actual del área, en los sectores bajo, medio y alto de la montaña predomina la cría de ganado, principalmente caprino, no siendo escasa la ovina, equinos, vacunos y mulares. La huerta familiar de los puestos ganaderos solo ocupa pequeños espacios de terrenos donde existen disponibilidad de agua y suelos como sucede también en sectores andinos análogos (Méndez, 2004, 2011). Otras actividades desarrolladas en el área son andinismo y el turismo aventura, entre otros. La trashumancia ganadera hacia los altos valles húmedos se realiza todos los años en verano; allí la vegetación de altura y particularmente de los sitos húmedos de las vegas o mallines localizadas en los cajones de los arroyos Chico, Pehuenche, Grande, entre otros, son aprovechados intensamente por el ganado durante las veranadas. $\mathrm{Al}$ respecto la población distingue dos épocas de pastoreo: las veranadas (en verano), en los campos de altura en la alta montaña y la invernada (en invierno) en las partes más baja del E, sobre el valle del río Grande desde Bardas Blancas hasta Las Loicas. Al respecto todos los años después de finalizar las veranadas se realiza una gran fiesta muy popular; conocida como: la Vuelta del Veranador ya incluida como una de las más importantes del departamento de Malargüe.

La población permanente del área es escasa y está concentrada en los valles. Los parajes más destacados son Bardas Blancas y Las Loicas. Además están los puestos distribuidos en los márgenes de arroyos y ríos. La acción antropogénica es bastante fuerte y avanza cada vez más manifestándose con sobrepastoreo, incendio, tala, entre otros, sobre las comunidades vegetales del área.

\section{Análisis de la vegetación}

Se utilizó el método fitosociológico de BraunBlanquet (1979) y se tomaron también algunas ideas, sobre todo en el ordenamiento de las tablas fitosociológicas, de Mueller-Dombois \& Ellenberg (1974). La labor de campaña se realizó entre los años 2006 y 2013 efectuando 470 relevamientos fitosociológicos. Estos relevamientos se clasificaron agrupándolos manualmente por su homogeneidad fisonómica, florística y ecológica, y fueron volcados en una tabla comparativa general de cuyo análisis surgieron 67 comunidades vegetales; de éstas solo19 fueron considerados como principales siendo distribuidas en 214 relevamientos seleccionados, teniendo en cuenta solo a las plantas vasculares. 
Con ésta base se construyeron tablas parciales y una tabla sintética de la vegetación (Tabla 1). Los relevamientos se realizaron en áreas mínimas de muestreo de: $100 \mathrm{~m}^{2}$ para matorrales, $25 \mathrm{~m}^{2}$ para pastizales, $1 \mathrm{~m}^{2}$ para vegas o mallines, 1 o más metros lineales para la vegetación de las grietas.

En este trabajo fueron analizadas e incluidas las comunidades edáficas de vegetación de las vegas o mallines, mientras que las saxícolas, ruderales, viarias, entre otros, no fueron consideradas en el presente trabajo. En todo momento se trató de graficar sobre las cartas topográficas las comunidades que podían ser factibles de representarlas a la escala de trabajo. Sobre la base de la homogeneidad fisonómica, florística y ecológica de las comunidades vegetales se realizaron los relevamientos fitosociológicos. Se observó y describió el aspecto fisonómico, florístico y ecológico de las unidades y se consideraron los dinamismos, las acciones antropogénicas y naturales que afectan a ellas. Se realizaron perfiles florísticos y se herborizaron varios ejemplares de las plantas de dudosa identificación a campo. Además se analizaron las formas biológicas (Raunkiaer, 1934) y corológicas (Cabrera, 1976).

Para la identificación de las especies se utilizaron principalmente las floras de Mendoza (Ruiz Leal, 1973; Roig 1972) y se consultaron y revisaron los especímenes del Herbario MERL. La nomenclatura de las plantas se hizo en base a las referencias del Catálogo de las Plantas Vasculares del cono sur de Sudamérica (Zuloaga et al., 2008a, b, c).

Para la cartografía de la vegetación se usaron fotografías aéreas 1:50.000 de la Dirección General de Fabricaciones Militares e imágenes satelitales del IGM, 1:250.000, y además imágenes del Google Earth.

\section{Análisis de datos}

La dispersión en el desarrollo altitudinal de las comunidades vegetales obtenidas, se hizo en función de una matriz formada por las temperaturas y altitudes (Tabla 3). Para ello se consideraron los valores promedios de altitudes obtenidos de los relevamientos y de temperaturas estimadas para cada una de las comunidades (19). Se aplicó la misma metodología de trabajos anteriores (Méndez, 2010, 2011) haciendo uso del análisis de dispersión y de conglomerados jerárquicos a través de Infostat (Di Rienzo et al., 2010). Se usó la forma euclidea y el método promedio (Average linkage) para graficar la dispersión y así obtener una mejor visualización de las relaciones y diferencias entre las comunidades vegetales.

\section{Análisis corológico}

Para categorizar corológicamente a las especies del área se usó la información de Cabrera (1976). Además se consultó bibliografía de otros autores (Soriano, 1956; Roig, 1960, 1989, Méndez et al., 1988; León et al., 1998; Martínez Carretero, 2004). Se utilizó, en la tabla sintética, letras mayúsculas para identificar las provincias fitogeográficas exclusivas Patagónica (P), Altoandina (A) y Monte (M) o una combinación de ellas (M/P, P/A, M/P/A) e incluso se consideraron a los elementos exóticos (X) (cosmopolitas, introducidas, naturalizadas, entre otras) siendo la mayoría malezas. De acuerdo con ello, los elementos típicamente Patagónicos (P) se encuentran en los sectores más bajos y medios, y los altoandinos (A) en los sectores altos de la montaña. Los del Monte (M) están débilmente representados.

\section{Resultados y Discusión}

Los pisos y las comunidades vegetales (Tabla 1)

La heterogeneidad de bioclima y los fuertes gradientes altitudinales entre Bardas Blancas y Paso Pehuenche, en las divisorias cordilleranas de los Andes, (aprox. de 1420 a 2800 m s.n.m., con un desnivel de más o menos $1400 \mathrm{~m}$, en aprox. $100 \mathrm{Km}$ ), posibilitan la existencia de diversas comunidades vegetales.

El análisis fitosociológico de la vegetación del área revela la presencia de comunidades vegetales diferenciadas fisonómica, florística y ecológicamente. La tabla sintética de relevamientos de las comunidades vegetales, ordenada en función de las altitudes y regiones fisiográficas, muestra a cada una de ellas diferenciadas por elementos característicos diagnósticos. En ella se destacan 19 comunidades, incluyendo a las vegas o mallines, y 24 grupos florísticos con especies características o diagnósticas identificadas con la letra $G$ y caracterizadas por valores de constancia en números romanos en la tabla sintética. Además la información se completa con: la ubicación de las Provincias Fitogeográficas, piso vegetal, altitud, 


\section{E. Méndez - Vegetación de los altos Andes Centrales}

comunidades, grupo y número de relevamientos de cada comunidad, y el número de especies. En lo posible se seleccionan los relevamientos más representativos de los estados más maduros o próximos a ellos.

En cada caso las comunidades vegetales en su ascenso altitudinal ingresan a los pisos superiores pero restringiéndose a áreas con condiciones ecológicas análogas a las de su propio piso. Entre los pisos vegetales existen áreas de ecotonos, zonas de transición entre dos unidades vecinas, que son cada vez más estrechas a medida que se asciende altitudinalmente hasta tener definidos sus límites (Fig. 2).

\section{Piso andino bajo patagónico}

Piso de los matorrales de Neosparton ephedroides, Larrea nitida, Colliguaja integerrima y Fabiana imbricata (1420 a 1600 m s.n.m.)

Comunidad de Neosparton ephedroides (Tabla 1, comunidad 1)

Esta comunidad se la observa fragmentariamente en el valle inferior del río Grande donde ingresa al oeste desde Bardas Blancas sobre los niveles de terrazas hasta los $1600 \mathrm{~m}$ s.n.m. Está también representada en proximidades del área, en las márgenes o isletas del $\mathrm{A}^{\circ}$ Agua Botada, un afluente del río Grande.

Es una comunidad riparia dominada por Neosparton ephedroides con ejemplares de hasta 3 $\mathrm{m}$ de alto y coberturas de 50 a $60 \%$ (Foto 1). Está sometida a los vientos que circulan por el valle del río Grande donde es común las remociones de arena y su acumulación al pie de las plantas donde se establecen especies anuales como Euphorbia klotzschii y Solanum euacanthum.

Tiene como especies características diagnósticas al Grupo G1 representados por Euphorbia klotzschii, Solanum euacanthum, Acaena splendens, Maihueniopsis glomerata, Rhodophiala mendocina y Maihuenia patagonica y está acompañada por las especies de los grupos G11 y G25. Es una comunidad pobre en especies; entre ellas figura con frecuencia aunque con débil cobertura Lecanophora ameghinoi, una caméfita herbáceo-leñosa. Tiene como especies de alta constancia a Mulinum spinosum y Tiquillia nuttallii que provienen de los matorrales de Chuquiraga oppositifolia y Adesmia volckmannii, vulgarmente conocida como"colimamil". Su contacto es la comunidad de Larrea nitida.

Comunidad de Larrea nitida (Tabla 1, comunidad 2)

Es una comunidad que aparece destacada en la base de las laderas con $10-15^{\circ}$ de pendiente y expuestas al norte. Se la observa fragmentariamente como manchas más oscuras y brillantes en las márgenes y terrazas del valle del río Grande. Sus ejemplares llegan hasta 2,50 m de alto y con coberturas del $80 \%$. Asciende desde Bardas Blancas, a 1420 m s.n.m., hasta un poco más al oeste del río Poti-Malal. Sus ramas apoyantes en el suelo acumulan arena y favorecen la entrada y establecimiento de Pappostipa chrysophylla, Junellia spathulata y Poa lanuginosa, fuertes indicadoras de la remoción de los sitios. Estas plantas y deposiciones de arena podrían servir de refugio y protección a la fauna del lugar. Con frecuencia aparece Schinus polygamus como acompañante.

La caracteriza el Grupo G2 con Larrea nitida, Lycium gilliesianum y Lecanophora ecristata y sus acompañantes principales son elementos de los grupos G3 y G11. Se contacta a mayor altura con la comunidad de Neosparton ephedroides, y por debajo y a modo de faja con el matorral de Colliguaja integerrima.

Comunidad de Colliguaja integerrima y Schinus polygamus (Tabla 1, comunidad 3 )

Esta unidad se presenta a modo de faja y también de modo fragmentario desde los 1460 a $1550 \mathrm{~m}$ s.n.m. en exposiciones norte y en pendientes de 15 a $35^{\circ}$.

Es una comunidad biestratificada con un estrato superior dominado por Colliguaja integerrima, arbusto resinoso, brillante con follaje verde más claro que el de Larrea nitida con ejemplares de hasta $2 \mathrm{~m}$ de alto y cobertura de hasta $90 \%$.

Tiene como especies características diagnósticas las del Grupo G3 con Schinus polygamus, Senecio covasii, Astragalus pehuenches y Proustia cuneifolia, de comportamiento ripario, entre otras especies. Es acompañada por especies del grupo G11.

Del mismo modo que la anterior este matorral retiene y acumula arena al pie de los tallos. Posee algunas malezas como Xanthium spinosum, Cirsium vulgare, Solanum triflorum, Lactuca serriola, etc. que indican la fuerte presión antrópica. Se contacta con Larrea nitida, Fabiana imbricata y Adesmia volckmannii -“colimamil". Tiene alta frecuencia Pappostipa chrysophylla. Hay facies de 
Schinus polygamus, Argylia bustillosii y Senecio filaginoides.

Esta comunidad ha sido señalada en otras áreas cordilleranas como las de los cordones montañosos del Plata y Portillo (Méndez, 2004, 2011).

Comunidad de Fabiana imbricata (Tabla 1, comunidad 4)

Está asociada a las terrazas de los cauces sobre todo del río Grande, particularmente en las grietas o fisuras de las rocas o de conglomerados donde las especies encuentran factores de compensación ecológica, similares a aquellos de los cauces de los ríos. Es una comunidad biestratificada con un estrato superior dominado por Fabiana imbricata, arbusto resinoso, de hasta $2 \mathrm{~m}$ de altura y coberturas de hasta $90 \%$.

La comunidad está caracterizada por el grupo diagnóstico G4 con Baccharis magellanica y Acantholipia seriphioides; sus acompañantes principales son los Grupos G11y G25.

Se contacta con los matorrales de Larrea nitida.

\section{Piso andino medio Andino-Patagónico}

Piso de los matorrales de Adesmia volckmannii, Chuquiraga oppositifolia, Adesmia pinifolia, Adesmia obovata (1500 a 2600 m s.n.m.)

Comunidad de Adesmia volckmannii con Schinus odonelli (Tabla 1, comunidad 5)

Se desarrolla en laderas con pendientes de 15 a $35^{\circ}$ y exposiciones $\mathrm{N}$ y S y en sitios llanos entre los 1500 y 1720 m s.n.m. en estribaciones del valle del río Grande desde casi Poti-Malal hasta aprox. Las Loicas. Es un matorral denso y extenso, frecuente en las terrazas aluviales del río Grande y $\mathrm{A}^{\mathrm{o}}$ Pehuenche.

Es una comunidad biestratificada dominada por Adesmia volckmannii con ejemplares de 1,50 a $3 \mathrm{~m}$ de alto y una cobertura variable entre 65 a $80 \%$. Vive en suelos arenosos con lapilli a veces con algunos clastos. Tiene fuerte presión del ganado, situación que favorece la presencia de malezas anuales nativas y exóticas como Xanthium spinosum y Erodium cicutarium, entre otras.

Está caracterizada por el grupo G5 representada, además de Adesmia volckmannii, por Jarava neaei y Mulguraea ligustrina de frecuente presencia y muy buscada por los animales aún dentro de las protección que le ofrecen las plantas de Adesmia volckmannii. Es común la presencia de trepadoras como Tropaeolum porifolium, Loasa argentina, Blumenbachia dissecta, entre otras. Schinus odonelli es frecuente y con elevados valores de cobertura. Además del grupo principal G5 crecen especies de los grupos G10, G11 y G25.

Las ramas de Adesmia volckmannii sufren el ataque de Pilostyles berteroi, frecuente en los matorrales presentes en el camino al valle Noble. Se pone en contacto con Colliguaja integerrima, Argylia bustillosii, Mulinum spinosum y Pappostipa speciosa, que probablemente lo reemplaza en el dinamismo de sucesión.

Comunidad de Chuquiraga oppositifolia (Tabla 1, comunidad 6)

Ocupa superficies considerables y a veces está en mosaico con otras comunidades. Se localiza entre los 1680 y 2310 m s.n.m., en sitios llanos y laderas expuestas al $\mathrm{N}$ y S con pendientes de hasta $30^{\circ}$. Es una comunidad biestratificada dominada por Chuquiraga oppositifolia con ejemplares de 0,50 a $1,80 \mathrm{~m}$ de alto y coberturas de 60 a $80 \%$.

Se extiende sobre suelos arenosos con lapilli y algunos clastos aislados.

Se caracteriza por contener a elementos del grupo G6 como Tropaeolum incisum, Pantacantha ameghinoi, Gutierrezia gilliesii, entre otros. Los principales grupos que lo acompañan son G10, G11 y G25. Tienen alta frecuencia Senecio subumbellatus, Poa holciformis, Hordeun comosum, Pappostipa speciosa, Junellia spathulata y Bromus setifolius var. setifolius.

Es una comunidad con fuerte presión de animales domésticos. Hay facies de Festuca thermarum entre 2000 y 2080 m s.n.m. Son comunes Senecio filaginoides, Junellia spathulata, Argylia bustillosii y Adesmia aegiceras. Tiene como contacto a distintas comunidades: la comunidad de Poa huecu en pastizales y vegas en el Cajón del río Chico o en las Termas del Cajón Grande, la de Adesmia pinifolia en la base de rocas aflorantes, la de Junellia spathulata, Cortaderia rudiuscula, Pappostipa speciosa y Festuca thermarum.

Comunidad de Pappostipa speciosa (Tabla 1, comunidad 7)

Es una comunidad extensa que se localiza entre los 1630 y 2240 m s.n.m. Vive generalmente en 


\section{E. Méndez - Vegetación de los altos Andes Centrales}

sitios de poca pendiente: casi llano, pero también en laderas de hasta $30^{\circ}$ aproximadamente expuestas al $\mathrm{N}$ y $\mathrm{S}$, en suelos arenosos con lapilli muy removidos.

Es dominada por Pappostipa speciosa con ejemplares de 0,30 a $0,50 \mathrm{~m}$ de alto y coberturas que van desde los $10 \%$ en áreas muy sobre pastoreadas hasta $80 \%$ en las más estabilizadas. La fisonomía de este pastizal se destaca por el color amarillo oro de sus matas.

Está caracterizada por especies del grupo G7 siendo sus principales elementos Montiopsis cistiflora, Adesmia corymbosa, Gayophyton micranthum y Boopis gracilis, entre otros. Sus contactos son: las comunidades de Adesmia obovata en altitud, de Panicum urvileanum, bien desarrollada como pastizal y de Adesmia volckmannii, camino al valle Noble, de Festuca gracillima, de Chuquiraga oppositifolia, de Festuca kurtziana, y de Mulinum spinosum en áreas muy degradadas y en toscales de relieves volcánicos. Los principales elementos que lo acompañan provienen de los grupos G10, G11 y G25.

Comunidad de Poa holciformis (Tabla 1, comunidad 8)

Se encuentra entre los 1720 y 2620 m s.n.m. Contiene elementos de la Patagonia como Adesmia volckmannii.

Poa holciformis caracteriza la comunidad y sus acompañantes principales son elementos de los grupos G10, G11 y G25. Los ejemplares de Poa holciformis tienen 0,30 a $0,60 \mathrm{~m}$ de alto y alcanzan coberturas de 40 a $80 \%$.

Es un pastizal de $30-50 \mathrm{~cm}$ de alto y coberturas de 30 a $80 \%$ que ocupa sitios llanos y de laderas con $25^{\circ} \mathrm{de}$ pendiente. Tiene como comunidades de contacto a las de Mulinun spinosum, Junellia uniflora y Chuquiraga oppositifolia.

\section{Comunidad de Adesmia pinifolia (Tabla 1, comunidad 9)}

Crece en forma fragmentaria en laderas expuestas hasta de $45^{\circ}$ de pendiente y sitios llanos entre los 1600 y 2346 m s.n.m.

La comunidad es biestratificada con ejemplares de Adesmia pinifolia de 0,50 a $3 \mathrm{~m}$ de alto y coberturas entre 30 y $75 \%$. Vive en suelos arenosos con lapilli.

Se caracteriza por el grupo de especies G8 representados por Adesmia pinifolia, Oenothera mendocinensis y Schizanthus grahamii. Los grupos principales que lo acompañan son G10, G11, G25. Tiene especies de alta constancia que le acompañan como Senecio filaginoides, S. subumbellatus, Mulinum spinosum, que forman facies, y también son frecuentes Junellia spathulata, Chuquiraga oppositifolia y Pappostipa speciosa. Por los relictos que todavía quedan Adesmia pinifolia debió conformar un paisaje de incomparable belleza en el área y sobre todo en el llano. Así se presenta en áreas de difícil acceso en la base de laderas o frentes de rocas aflorantes, como en Las Loicas y particularmente en toscales camino al valle del Noble donde se registran, antes del paraje de la Invernada, ejemplares de hasta 3,50 m de alto. El matorral de Adesmia pinifolia está presionado por el pastoreo y esto lo denuncia la presencia de elementos exóticos como Cirsium vulgare y Salsola kali, entre otros. Hay facies de Chuquiraga oppositifolia y Junellia spathulata, apareciendo también Berberis empetrifolia.

$\mathrm{Al}$ respecto es un área de fuerte densidad de animales en las veranadas y no sería extraño que antes de la entrada del hombre blanco, incluso aborigen, todo fuera ocupado por Adesmia pinifolia. Es una comunidad fuertemente presionada por el hombre y los animales. El dinamismo hacia su mayor degradación podría ser:

Adesmia pinifolia - Pappostipa speciosa

La fisonomía del matorral se asemeja a la hallada en el Parque Provincial Aconcagua y Cordón del Portillo (Méndez, et al., 2006; Méndez, 2011). Sus contactos son las comunidades de Adesmia volckmannii, Chuquiraga opposifitolia y Argylia bustillosii.

Comunidad de Adesmia obovata (Tabla 1, comunidad 10)

Está en sitios llanos o laderas con exposición $\mathrm{S}$ y N y pendientes de 10 a $30^{\circ}$ entre $\operatorname{los} 2180$ y 2360 m s.n.m.

Es una comunidad biestratificada dominada por A. obovata con ramas marrones y brillantes de 1,50 a $1,70 \mathrm{~m}$ de alto y coberturas de 60 a $80 \%$. Está acompañada con alta frecuencia por Poa holciformis, Senecio subumbellatus, Junellia spathulata y Mulinum spinosum.

Los suelos son arenosos con lapilli y algo de arcilla y en laderas con gravillas y rodados. 
Se caracteriza por el grupo G9, con Adesmia obovata, y Tweedia aucaensis. Los principales acompañantes son elementos de los grupos G10, G11y G25.

Es una comunidad fuertemente pastoreada con elementos que lo denuncian como Junellia spathulata, Mulinum spinosum, Acaena pinnatifida y Seneciofilaginoides. Sus contactos más frecuentes son las comunidades de Berberis empetrifolia y Pappostipa speciosa.

\section{Piso andino alto Andino-Altoandino}

Piso de las estepas de Azorella monantha, Nassauvia revoluta, Empetrum rubrum $y$ Ochetophila nana (2500 a 2800 m s.n.m.)

Comunidad de Azorella monantha (Tabla 1, comunidad 11)

Se encuentra por encima de los 2600 m s.n.m., en los sitios planos altoandinos con suelos de arenas volcánicas y lapilli más o menos compactados y con nieve más permanente. Su aspecto es de una estepa en cojín placa de menos de $0,10 \mathrm{~m}$ de alto y coberturas de la comunidad de 5 a $20 \%$.

Se caracteriza por el Grupo12 con especies diagnósticas como Chaetanthera villosa, Mulinum crassifolium, Carex aff. patagonica, entre otras. Azorella monantha forma cojines en placas, compactos y durísimos. Sus principales acompañantes son especies del grupo G24.

En la base de laderas tiene su contacto con Poa holciformis y Mulinum spinosum. Sus principales acompañantes son Lecanophora cframeghinoi, Moschopsis monocephala y Pozoa coriacea, mimética y con débil cobertura.

Comunidad de Nassauvia revoluta (Tabla 1, comunidad 12)

Se documenta entre 2500 y 2560 m s.n.m., en laderas de suave pendientes de hasta $15-20^{\circ}$ expuestas al SSE en el piso altoandino.

Está dominada por una estepa de Nassauvia revoluta con ejemplares de 0,05 a $0,30 \mathrm{~m}$ de alto $\mathrm{y}$ coberturas de 5 a $35 \%$, con hojas grises, recurvadas y mimética en los suelos de lapilli volcánico. Es común en el Paso Pehuenche y aparece a veces semienterrada en la arena de pómez del granulado volcánico.

Se caracteriza por la presencia del grupo de especies G13 con elementos diagnósticos con alta frecuencia como Trisetum preslei, Acaena macrocephala, Adesmia spuma, Quinchamalium chilense, entre otras. Sus acompañantes son elementos de los grupos G20 y G24. En replanos de suelos arenosos con pumicitas y clastos pequeños de lapilli, aparecen facies de Oxalis adenophylla cuyos ejemplares miden 0,10 a $0,30 \mathrm{~m}$ de alto $\mathrm{y}$ sus coberturas alcanzan hasta el $40 \%$. Esta especie tiene raíces bulbo-tuberosas escamosas y es de fácil reconocimiento por sus vistosas flores lilacinas. Se contacta con las estepas de Empetrum rubrum o vegas de Ranunculus peduncularis.

Comunidad de Empetrum rubrum (Tabla 1, comunidad 13)

Se destaca por su fisonomía, a modo de manchas oscuras, entre los 2500 y 2600 m s.n.m. Ocupa suelos arenosos con materia orgánica y húmedo en replanos o laderas de suaves pendiente de 10 a $15^{\circ}$ con exposiciones al N. Es una estepa dominada por Empetrum rubrum de 0,05-0,10 m de alto y coberturas de hasta $100 \%$.

Tiene como elementos característicos diagnósticos al grupo G14 representada por Agrostis imberbis, Cerastium aff. arvense, Pernettya prostrata, Chiliotrichum diffusum, entre otros. Son elementos acompañantes algunas especies de los grupos G15, G16, G18, G20 y G24. Sus contactos son las comunidades de Ranunculus peduncularis, Nassauvia revoluta, Ochetophila nana y hasta Rumex acetosella y Patosia clandestina. Tiene facies de Pernettya prostrata, Luzula racemosa, Agrostis imberbis y Chiliotrichum diffusum. Forma parte de la tundra andina.

Comunidad de Ochetophila nana (Tabla 1, comunidad 14)

Se presenta en pequeñas superficies rocosas avanzando sobre ellas a modo de carpetas o alfombras. También está en el piso altoandino superior entre 2560 y 2600 m s.n.m. Vive en suelos arenosos, ripioso con lapilli y con materia orgánica.

Forma una estepa o carpeta densa de 0,05-0,10 $\mathrm{m}$ de alto y coberturas de hasta el $100 \%$ que serviría de refugio a la fauna de la zona.

Tiene especies características diagnósticas presentes en el grupo G15 a Montiopsis aff. gayana, Werbebauera colchaguensis, Gamocarpha gilliesii, entre otras. Tiene como acompañantes principales especies de los grupos G16, G20 y G24. Su comunidad de contacto es la de Patosia clandestina. 
Vegetación de las vegas o mallines

En general es posible diferenciar fisonómica, florística y ecológicamente a las vegas de altura y de baja altura.

\section{Vegas de altura}

Comunidad de Ranunculus peduncularis (Tabla 1, comunidad 15)

También está asociada a los cursos de agua como fajas o manchas húmedas de sitios ricos en materia orgánica con arena y algo de arcilla. Ranunculus peduncularis forma una carpeta herbácea densa de 0,05-0,20 m de alto y coberturas de hasta el $100 \%$

La caracterizan especies del grupo G17 con elementos diagnósticos como Geranium aff. sessiliflorum, Plantago barbata, Diplolepis nummulariifolia, Olsynium aff. junceum, entre otros. La acompañan elementos de los grupos G20, G23 y G24. Tienen mediana frecuencia Luzula racemosa y Plantago barbata. Sus contactos más frecuentes son las comunidades de Patosia clandestina y Empetrum rubrum.

\section{Comunidad de Patosia clandestina (Tabla 1,} comunidad 16)

Es una comunidad destacada fisonómicamente por sus cojines densos de distintos tamaños que usufructúan la humedad de las vegas o mallines de altura por encima de los 2200 m s.n.m., hasta más de $2600 \mathrm{~m}$ s.n.m. Está en las nacientes de los arroyos y ríos cordilleranos tanto en el Cajón Grande, Chico como del Pehuenche y en la gran base del $\mathrm{C}^{\circ}$ Campanario. Con frecuencia ocupa sitios planos pero también las bases de laderas, siempre con el agua casi en superficie y en suelos orgánicos ricos en arenas.

Los cojines de Patosia clandestina son de 0,40-0,50 m de alto y algunos superan los $2 \mathrm{~m}$ de diámetro. Sobre la superficie del mismo se destacan Festuca magellanica, Deschampsia caespitosa, Gentianella magellanica, Poa pratensis, Valeriana carnosa, entre otras, que viven en los espacios abiertos del cojín aprovechando la humedad y nutrientes de los suelos orgánicos. Estas plantas son engullidas por el crecimiento del cojín y muertas en una situación similar a lo observado en otras áreas (Méndez, 2011). Al respecto todas estas plantas que viven en el cojín también lo hacen normalmente en los espacios entre cojines y también en los bordes de los cursos de los arroyos con agua permanente, ya sea formando pequeñas comunidades o facies dentro de otras comunidades.

Está caracterizada por especies del grupo G18, entre otros Festuca magellanica, Gentianella magellanica y Gentiana prostrata. Otras especies acompañantes son elementos de los grupos G20, G23 y G24. Las comunidades de contacto son las de Ochetophila nana, Empetrum rubrum, Pernettya pumila, Ranunculus peduncularis $y$ Festuca kurtziana.

Comunidad de Festuca kurtziana (Tabla 1, comunidad 17)

Se localiza por encima de los 1900 m s.n.m., en los bordes de los cursos de los arroyos con agua permanente de los valles y laderas de sitios húmedos y humíferos con arena y materia orgánica. Es una comunidad con un color verde azulado dominada por Festuca kurtziana con ejemplares de hasta $0,60 \mathrm{~m}$ de alto y coberturas de 75 a $90 \%$. Ocupa suelos arenosos algo arcillosos tanto en sitios llanos como laderas.

Se caracteriza por contener elementos característicos diagnósticos como Carex patagonica, Vicia pampicola, Deschampsia patula, Azorella trifurcata, entre otras. Hay facies de Rumex acetosella y tiene alta constancia Juncus balticus. Son acompañantes además elementos de los grupos G20, G23 y G24.

Es común a lo largo de las márgenes de los arroyos donde se enriquece con elementos de las diferentes comunidades con las que se contacta, como las de Senecio subumbellatus en áreas muy alteradas, Berberis empetrifolia, Juncus balticus, Pappostipa speciosa, Carex gayana, Chuquiraga oppositifolia, Patosia clandestina y Junellia spathulata.

\section{Vegas de baja altura}

Comunidad de Juncus balticus (Tabla 1, comunidad 18)

Se localiza desde Bardas Blancas hasta las Loicas, entre los 1420 y 1720 m s.n.m., respectivamente, y a lo largo de los bordes de los arroyos y Río Grande y en sus vertientes o laderas. Allí ocupa suelos orgánicos y humíferos con arena.

Tiene ejemplares de 0,10 a $0,40 \mathrm{~m}$ de alto y coberturas de hasta el $100 \%$. Son elementos 
característicos diagnósticos el grupo G21 representado por Eleocharis pseudoalbibracteata, Polypogon monspeliensis, Veronica anagallis-aquactica, entre otros. Contiene elementos introducidos como Cirsium vulgare, Trifolium repens, Cotula coronopifolia, Chenopodium ambrosioides y Sonchus oleraceus indicadores de la fuerte presión del pastoreo y de la actividad humana. Vive en suelos arenosos, humíferos, húmedos o con freática superficial. Tiene como acompañantes principales a elementos de los grupos G23 y G24. Sus contactos más frecuentes son la comunidad de Cortaderia rudiuscula y Fabiana imbricata en las terrazas del río Grande y proximidades de Bardas Blancas.

\section{Comunidad de Cortaderia rudiuscula (Tabla 1, comunidad 19)}

Está representada en los márgenes de los ríos y arroyos desde Bardas Blancas hasta proximidades de las Loicas para luego ser observada en las márgenes de los arroyos la Matancilla, Cajones del río Chico, Grande, en vertientes húmedas de laderas en suelos arenosos con materia orgánica y algo salinos. Su fisonomía es la de un pajonal o cortaderal con ejemplares de Cortaderia rudiuscula de hasta 2, $50 \mathrm{~m}$ de alto y máximas coberturas. Se caracteriza por la abundancia de malezas.

Son características diagnósticas elementos del grupo G22 y se destacan Baccharis salicifolia, Muhlenbergia asperifolia, Lathyrus latifolius, entre otros. También lo acompañan elementos de los grupos G23 y G24. Sus contactos son las vegas como las de Juncus balticus, y matorrales de Chuquiraga oppositifolia por encima de ella o de Junellia spathulata y Mulinum spinosum en niveles de terrazas de cauces de arroyos y ríos.

Es una comunidad indicadora de sitios húmedos de márgenes de los arroyos y ríos, también ocupa las vertientes húmedas de laderas siguiendo los cursos de aguas.

\section{Los pisos de vegetación}

En el área es posible diferenciar los pisos de vegetación según altitudes y condiciones termopluviométricas de modo semejantes a otros sitios ya estudiados como en el Parque Aconcagua, Portillo, del Plata, etc. (Méndez, 2004, 2010, 2011).

En función del relieve y altitudes de esta montaña se diferencias 3 grandes sectores: al W el más alto, con la montaña propiamente dicha, al E el más bajo y uno intermedio en situaciones medias de montaña.

Del análisis general de las comunidades vegetales del área se revela la existencia de 24 grupos florísticos de especies donde 4 son exclusivos del sector Andino inferior (comunidades 1 a 4), 6 del Andino medio (comunidades 5 a10), y 4 del Andino superior (comunidades 11 a 14). Son representantes de las Vegas 2 pisos: de altura (comunidades 15 a 17) y de baja altura (comunidades 18 y 19). El grupo G24 es el de mayor amplitud ecológica y acompaña a todos ellos .

\section{Análisis corológico}

El área analizada está representada por comunidades enriquecidas, la mayoría de ellas, por elementos Patagónicos y Altoandinos (Tablas 1, 4, 5). En la Tabla 4 se aprecia la distribución de los elementos corológicos exclusivos; la mayor cantidad de elementos de las comunidades inferiores son de carácter patagónico $(\mathrm{P}), \operatorname{los} \mathrm{de}$ las comunidades superiores, y que dominan el sector más alto de la montaña, son altoandinos (A) como también las de las vegas de altura. En una situación intermedia aparecen elementos patagónicos (P) y la combinación patagónico andino $(\mathrm{P} / \mathrm{A})$ particularmente en el sector medio de la montaña. Esta última también tiene elementos (P/A) que igualmente acompañan al sector altoandino representados por elementos exclusivos.

La mayor amplitud de elementos corológicos se hallan en ambientes de las vegas de baja altura donde se equilibran sobre todo en la comunidad de Cortaderia rudiuscula, y existe una fuerte cantidad (14) de elementos de más amplia combinación (M/P/A) en la comunidad de Festuca kurtziana.

En la Tabla 5 se observa que sobre el total de especies en el área (230) las dos provincias fitogeográficas dominantes son la Provincia Patagónica (P) y la Altoandina (A).

En el piso vegetal bajo (piso 1) los elementos patagónicos (20) están presentes en un 35,1\% y en el piso vegetal alto (piso 3 ) los elementos altoandinos (70) alcanzan el 77,8\%, aproximadamente, del total de elementos en cada piso. En el piso vegetal medio (piso 2), en sectores medios entre estas principales categorías dominantes, aparecen combinaciones de P/A (36) que representan aproximadamente el $36,7 \%$ del total de éste piso. 


\section{E. Méndez - Vegetación de los altos Andes Centrales}

Con respecto a las vegas de altura se caracterizan por los elementos altoandinos (41) presentes en un $62,1 \%$, mientras que las de baja altura solo la combinación de P/A (8) alcanza el 28,6\% y ello posiblemente obedezca, por las fluctuaciones climáticas, al descenso o ascenso de las especies hacia esos sectores.

Por otro lado las plantas exóticas (X) son abundantes en las vegas de baja altura (12) con un $42,8 \%$ de ellas, a consecuencia de las mayores presiones antrópicas que allí se producen. Son menos abundantes en las vegas de altura (7) donde solo se presentan un $10,6 \%$.

En el área estudiada las altitudes de las comunidades vegetales disminuyen latitudinalmente a medida nos dirigimos al sur y, por lo contrario aumentan hacia el norte. El sector más alto de la montaña está representado por la Provincia Altoandina, y el sector más bajo por la Provincia Patagónica, ambos dentro del Dominio Andino-Patagónico y posiblemente identificadas por el distrito Nor Patagónico y la Provincia Altoandina -Patagónica, respectivamente. El área estudiada tendría al piso Altoandino representado fragmentariamente por las comunidades de Azorella monantha, y Nassauvia revoluta las que poseen especies comunes a las relevadas en otras latitudes en el sector altoandino (Méndez, 2004, 2010, 2011). Por otro lado a altitudes por encima de los 2800 m s.n.m., éstas comunidades tal vez podrían estar representadas, también fragmentariamente y cartográficamente, como islas a o islotes en proximidades de los cerros Trolón, de la Ventana, el Callao y Campanario. Probablemente estas comunidades de Azorella monantha y Nassauvia revoluta se contacten más arriba con la comunidad de Adesmia subterranea (no relevada en este trabajo).

Se señala que el reemplazo de Larrea divaricata y Condalia microphylla sería un indicador del paso de la provincia del Monte a la Patagónica (Cabrera, 1976) y esta sentencia para el área se cumple porque estas especies no se encuentran en la misma.

Según León et al. (1998) destacan para la Provincia Patagónica, entre otros distritos, al Distrito Occidental que se localiza al sur del paralelo $38^{\circ}$, fuera de los límites de nuestra área, y en donde, en las comunidades más representativas de la estepa arbustiva-graminosa, citan a especies comunes o coincidentes que se encuentran en ella, tales como Stipa speciosa (=Pappostipa speciosa), Adesmia campestris (=A. volckmannii), Poa lanuginosa, Senecio filaginoides, Mulinum spinosum, Schinus polygamus, Bromus setifolius, Hordeum comosum, Perezia recurvata, Nassauvia axillaris, Grindelia chiloensis, Colliguaja integerrima, Lecanophora ameghinoi, Chuquiraga oppositifolia, Verbena ligustrina (=Mulgaraea ligustrina) y Poa ligularis. También señalan al Distrito de la Payunia, ya considerado por Cabrera (1976), donde dan elementos representativos como Mulinum spinosum, Ephedra ochreata, Colliguaja integerrima, Berberis grevilleana y Astragalus pehuenches. Además registran al Monte-patagónico con especies como Larrea nitida, Senecio filaginoides, Mulinum spinosum, Panicum urvilleanum y Acantholippia seriphioides también presentes en el área considerada.

Por otro lado, Martínez Carretero (2004) da a conocer, dentro del Dominio Andino Patagónico, a la Provincia Fitogeográfica de la Payunia, en contacto al $\mathrm{E}$ con el área $\mathrm{y}$, donde incluye algunas comunidades o asociaciones comunes como de Adesmia pinifoliae, Colliguayetum integerrima, Mulinum spinosi, bajo un bioclima semiárido superior. Al respecto señala en ella a algunos elementos patagónicos como Neosparton ephedroides, Grindelia chiloensis, Pantacantha ameghinoi, presentes en área y hasta la asociación Poetum durifoliae localizada con un bioclima subhúmedo en la unidad fitogeográfica Altoandino. Esta asociación no fue encontrada en nuestra área. Así del análisis de la tabla sintética comparativa de las 19 las comunidades vegetales de la Payunia (Martínez Carretero, 2004), se revela que solo algunos matorrales, que considera patagónicos, tienen semejante fisonomía a los del área estudiada, entre ellos, el Adesmietum pinifoliae, donde incluye, además de Adesmia pinifolia, algunos elementos comunes como Chuquiraga oppositifolia, Pantacantha ameghinoi, Argylia bustillosii, Phacelia secunda y Malesherbia lirana. Otro tanto ocurre con el Retanillo-Colliguayetum, parcialmente representada en el área por las especies comunes de Colliguaja integerrima, Grindelia chiloensis, Ephedra ochreata, Nassauvia axillaris y Junellia spathulata. Seguramente la presencia de todos estos elementos en nuestra área se relacione con una débil entrada de los mismos por los sectores más bajos del Valle del río Grande. 
Cabe recordar que las fisonomías pueden enmascarar la realidad. Así la fisonomía del matorral de Larrea nitida puede responder a la del Monte, pero estructuralmente en su composición florística dominan los elementos patagónicos, en una situación semejante a la que documentara Ruiz Leal en su trabajo sobre el Rincón del Lirkay o en la Meseta de Somuncurá (Ruiz Leal, 1961, 1972). Por ello en este trabajo se sigue el criterio de considerarlas según nuestro análisis ubicadas dentro de la Provincia Patagonica. Todo este análisis nos induce a separar elementos que son exclusivos de Patagonia (P) y Altoandino (A) y que se considere la combinación de ellas $(\mathrm{M} / \mathrm{P}, \mathrm{P} / \mathrm{A}$, $\mathrm{M} / \mathrm{P} / \mathrm{A})$. Tal vez en el futuro haciendo uso de toda la información disponible y la aplicación de los complejos de vegetación se consiga alcanzar y dar mayor claridad a éstos tratamientos fitogeográficos.

\section{Las relaciones con las formas de vida}

En general existe una buena correspondencia de las formas de vida en la distribución de las comunidades vegetales en el gradiente altitudinal del área estudiada (Tabla 2). En función de ellas también puede definirse a las condiciones bioclimáticas de las comunidades o pisos vegetales. Así las comunidades del sector andino bajo (1 a 4) se caracterizan por el dominio de terófitas que ponen en evidencia las condiciones más cálidas y secas de este ambiente.

Las condiciones media más atemperadas y menos secas se presentan en los relieves medios de la montaña donde las comunidades (5 a 10) que se asemejan entre sí, están dominadas por terófitas, hemicriptófitas y caméfitas indicando condiciones más secas y cálidas del sector bajo de la montaña y una mayor antropización, sobre todo por el notable incremento de las terófitas. Precisamente este sector está muy sobrepastoreado.

Las condiciones frías y húmedas del sector altoandino, representado por las comunidades (11 a 14) se caracteriza por el dominio de hemicriptófitas y caméfitas. Estas condiciones también se hallan en las comunidades de las vegas de altura (15 a 17) donde dominan las caméfitas, hemicriptófitas y geófitas, mostrando el bioclima camefítico y hasta situaciones más atenuadas de bioclima hemicriptofitico con los pastizales de Festuca kurtziana (17). Igualmente las comunidades de las vegas de baja altura $(18,19)$ están más equilibradas y con una tendencia de bioclima terofiticohemicriptofítico con mayores exposiciones a calores estivales y con fuerte reducción del número de especies.

\section{Las relaciones con las variables ambientales}

Los factores termopluviométricos cumplen un papel importante en la adaptación de las especies y comunidades vegetales y ellos pueden definir la presencia o no de las especies, comunidades y pisos vegetales que se desarrollan en el rango altitudinal (Méndez, 2010, 2011).

Los resultados de la dispersión en el espacio de las 19 comunidades vegetales consideradas muestran en función de los valores promedios de las temperaturas y altitudes, que tanto los gráficos de dispersión (Fig. 3) como de clasificación (Fig. 4) son equivalentes y señalan la existencia, a un nivel de distancia euclidea alta, a dos grandes grupos florísticos de comunidades que se corresponden con la Patagonia (1 a 9) y Altoandino (10 a 17) y a un nivel menor a 5 grupos de comunidades: 3 en el altoandino (12-16, 11 y 10-17) y 2 de la Patagonia (1-4 y 5-9) incluido las vegas de baja altura (18 y 19). Se puede ver que las comunidades de 1 a la 4, son muy similares entre sí y muy diferentes al resto de las comunidades. Además el piso medio andino es más similar al piso altoandino brindando evidencia a favor de la nomenclatura utilizada para clasificar los tres pisos de vegetación. Por otra parte parecería haber una gran heterogeneidad interna entre el piso andino y piso altoandino. Así en el primero claramente la comunidad 5 se relaciona en su composición a las comunidades de las vegas de baja altura 18 y 19, mientras que las otras se diferencian de estas y se relacionan mucho entre sí. En el piso altoandino, las comunidades de vegas de altura 15,16 y 17 parecen ser muy diferentes entre sí al igual que las comunidades 10,11 y el conjunto 12,13 y 14 , que está muy emparentado con comunidad 15 de la vega de altura. Por la fuerte correspondencia entre aquellas variables con las comunidades separando grupos de comunidades, de la Patagonia y Altoandina, no fue necesario ajustar el orden de la Tabla 1, la que se mantuvo. Estas relaciones se asemejan a los obtenidos en otras latitudes más al Norte (Méndez, 2004, 2010, Roig 1998). 


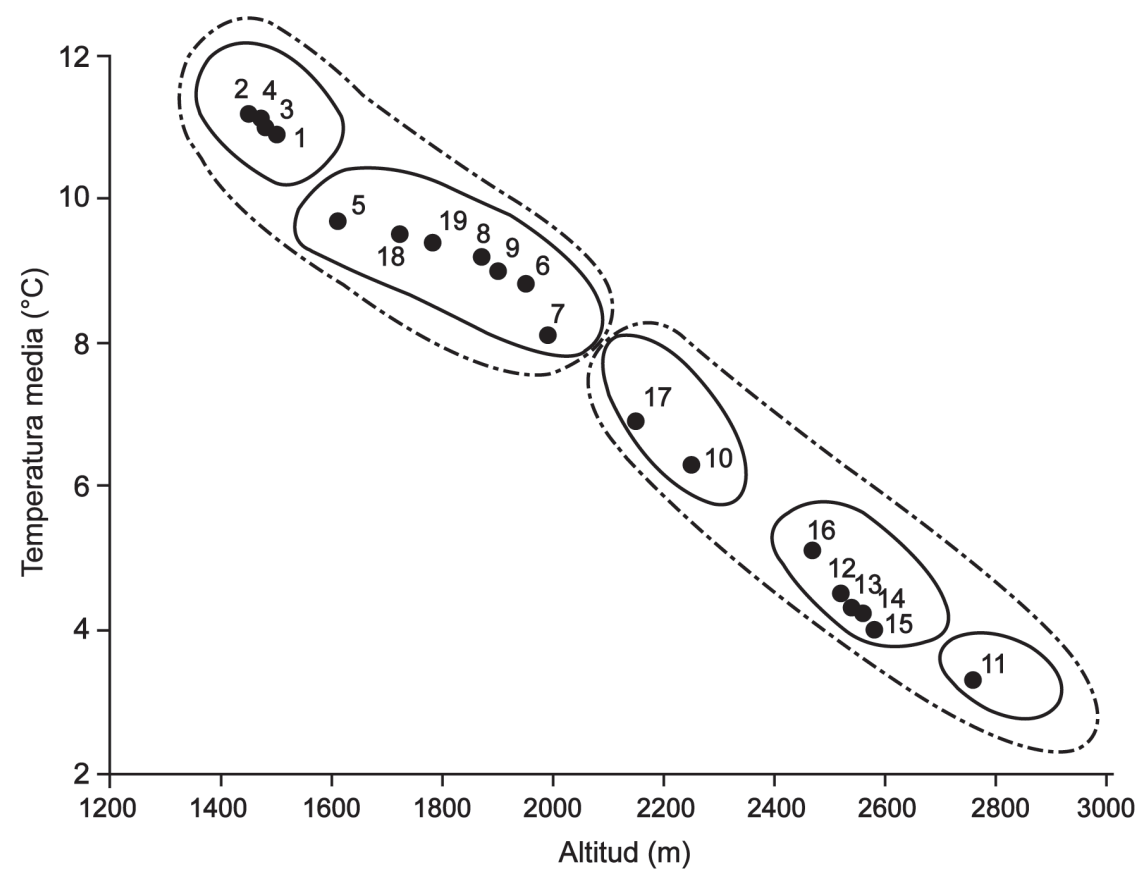

Fig. 3. Diagrama de dispersión de las comunidades vegetales de Bardas Blancas-Paso Pehuenche.

\section{Las relaciones florísticas con otras áreas}

Las comunidades vegetales del área en el gradiente altitudinal del área considerada de Bardas Blancas- Paso Pehuenche en el Sur mendocino, están poco relacionadas principalmente en el sector altoandino con las del Cordón del Plata y del Portillo (Méndez, 2004, 2011) donde se presentan muy pocas de las comunidades y su flora, e igualmente sucede con las comunidades desarrolladas en sectores intermedios y bajos de montaña donde solo figuran algunas de aquellas especies señaladas por Martínez Carretero (2004) y hasta esporádicamente presentes en otros trabajos bajo condiciones análogas (De Marco et al., 1993). Estas relaciones se van haciendo cada vez más lejanas hacia el norte por el ingreso franco de otras unidades y elementos fitogeográficos, como los de la Puna en el norte de Mendoza (Roig \& Martínez Carretero, 1998), o en el NW de San Juan donde se destaca la presencia solo de algunas de sus especies.

Por otro lado, el mapa bioclimático desarrollado por Martínez Carretero (2004, fig. 4) incluye todo el sector dentro del bioclima semiárido no desarrollando la parte altoandina que se corresponde a uno subhúmedo o a lo sumo semiárido superior. Al respecto considero que las comunidades halladas en este trabajo pertenecen al Dominio Andino Patagónico, a la Provincia Patagónica y a la Provincia Altoandina. La razón probablemente de mantener la clasificación de las provincias fitogeográficas de Cabrera (1976) obedecería al análisis de la distribución corológica de las especies y de las comunidades en su desarrollo altitudinal incluyendo hasta la montaña todo lo cual nos parece que permiten tener una idea más acabada de la corología de la especies y de sus asociaciones en el contexto fitogeográfico del área estudiada.

\section{ConClusión}

La vegetación de la transecta Bardas BlancasPaso Pehuenche, Malargüe, Mendoza, Argentina, en un gradiente altitudinal de $1420 \mathrm{~m}$ s.n.m a aprox. $2800 \mathrm{~m}$ s.n.m., se revela con el análisis fitosociológico de 19 comunidades vegetales. Estas se caracterizan por especies diagnósticas, exclusivas, preferenciales y acompañantes que permiten identificarlas y separarlas en 24 grupos florísticos. 
Bol. Soc. Argent. Bot. 49 (2) 2014

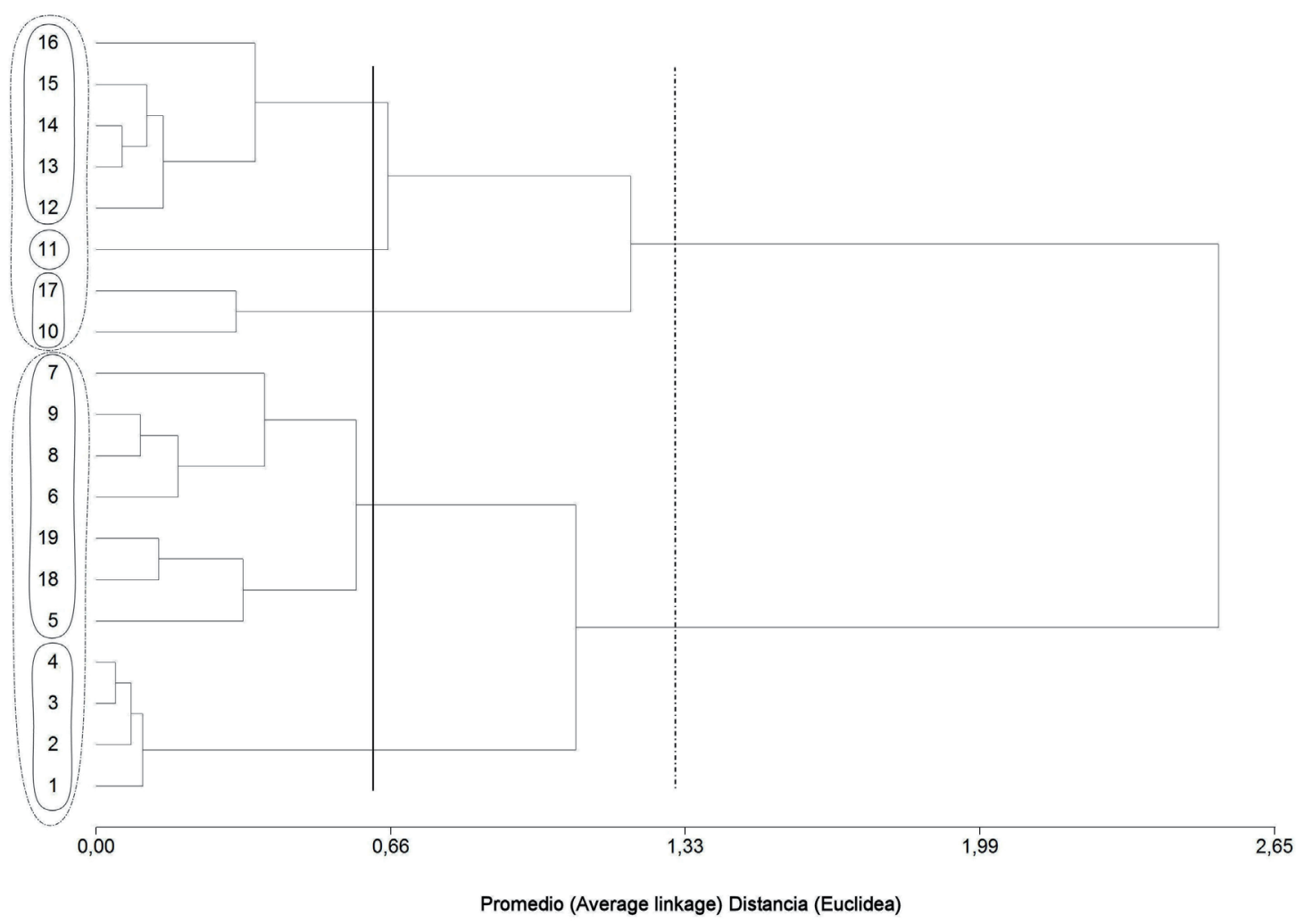

Fig. 4. Dendrograma de las comunidades vegetales de Bardas Blancas-Paso Pehuenche. Análisis de conglomerados jerárquicos.

Hay una relación florística-ecológica con los ambientes fitogeográficos y las formas de vida de las especies desarrolladas en el gradiente de altitud del área analizada.

El gradiente de altura, paralelamente con sus características climáticas, condicionan la composición florística de cada una de las comunidades vegetales del área. El análisis de dispersión y de conglomerados jerárquicos, permite reafirmar las estrechas relaciones de las comunidades vegetales con las temperaturas y altitudes. El estudio corológico revela la presencia dominante de las Provincias Fitogeográficas Patagónica y Altoandina que se corresponden con 3 pisos de vegetación reconocidos fitosociológicamente.

$\mathrm{El}$ recorrido altitudinal de $\mathrm{E}$ a W muestra distintos pisos de vegetación:

\section{Piso andino bajo Patagónico}

I. Piso de los matorrales de Neosparton ephedroides, Larrea nitida, Colliguaja integerrima y Fabiana imbricata 1420 a1600 m s.n.m.

\section{Piso andino medio Andino-Patagónico}

II. Piso de los matorrales de Adesmia volckmannii, Chuquiraga oppositifolia, Adesmia pinifolia, Adesmia obovata .1500 a 2600 m s.n.m.

\section{Piso andino alto Andino-Altoandino}

III. Piso de las estepas de Azorella monantha, Nassauvia revoluta, Empetrum rubrum $y$ Ochetophila nana .2500 a 2800 m s.n.m.

Todos estos pisos pueden ser factibles de extrapolarse al norte y más probablemente al sur del área estudiada. Independientemente de poder realizar en un futuro un análisis más profundo de la flora del área el análisis de la tabla sintética revela la presencia de 230 especies distribuídas en 176 géneros y 60 familias de plantas. Asteraceae y Poaceae son las más representativas con 29 y 18 géneros respectivamente. 
E. Méndez - Vegetación de los altos Andes Centrales

Tabla 1. Comunidades vegetales principales de Bardas Blancas-Paso Pehuenche (Malargüe, Mendoza). Comunidades vegetales: 1-19, Grupos florísticos G1-G24. Valores de constancia de las especies en cada comunidad (Números romanos) : I(<20), II(20-40), III(40-60), IV(60-80), V(>80).

\begin{tabular}{|c|c|c|c|c|c|c|c|c|c|c|c|c|c|c|c|c|c|c|c|c|c|}
\hline \multirow{3}{*}{\begin{tabular}{|l} 
Provincia fitogeográfica \\
Piso vegetal de:
\end{tabular}} & \multicolumn{10}{|c|}{ Patagónica } & \multirow{2}{*}{\multicolumn{4}{|c|}{$\frac{\text { Altoandina }}{3}$}} & \multicolumn{5}{|c|}{ Vegas de: } & \multirow[t]{3}{*}{ A } & \multirow[t]{3}{*}{ B } \\
\hline & \multicolumn{4}{|c|}{1} & \multicolumn{6}{|c|}{2} & & & & & \multicolumn{3}{|c|}{4} & \multicolumn{2}{|c|}{5} & & \\
\hline & & & & & & & & & & & & & & & & altura & & & uja & & \\
\hline Altitud m s.n.m. $(x=10 \mathrm{~m})$ & \multicolumn{4}{|c|}{$141-160$} & \multicolumn{6}{|c|}{$150-260$} & $>280$ & \multicolumn{3}{|c|}{$250-280$} & \multicolumn{3}{|c|}{$220-260$} & & -200 & & \\
\hline Comunidades $\mathrm{N}^{\circ}$ : & 1 & 2 & 3 & 4 & 5 & 6 & 7 & 8 & 9 & 10 & 11 & 12 & 13 & 14 & 15 & 16 & 17 & 18 & 19 & & \\
\hline Grupos de relev. No: & 3 & 3 & 3 & 3 & 4 & 4 & 4 & 3 & 4 & 4 & 2 & 4 & 4 & 4 & 2 & 2 & 2 & 2 & 2 & & \\
\hline Número de relev.: & 6 & 5 & 10 & 13 & 20 & 26 & 25 & 10 & 17 & 8 & 5 & 12 & 12 & 4 & 6 & 10 & 13 & 6 & 6 & & \\
\hline Número de especies: & 22 & 14 & 29 & 29 & 48 & 66 & 50 & 34 & 48 & 32 & 16 & 47 & 48 & 34 & 23 & 42 & 28 & 19 & 16 & & \\
\hline Especies características & diag & ósti & & & & & & & & & & & & & & & & & & & \\
\hline $\mathrm{G} 1$ & & & & & & & & & & & & & & & & & & & & & \\
\hline $\begin{array}{l}\text { Neosparton } \\
\text { ephedroides }\end{array}$ & V & . & . & . & . & . & . & . & . & . & . & . & . & . & . & . & . & . & . & $\mathrm{N}$ & $\mathrm{P}$ \\
\hline Euphorbia klotzschii & IV & II & 1 & . & . & . & . & . & . & . & . & . & . & . & . & . & . & . & . & $\mathrm{T}$ & $M / P$ \\
\hline Solanum euacanthum & II & . & . & . & . & . & . & . & . & . & . & . & . & . & . & . & . & . & . & T & $\mathrm{M} / \mathrm{P}$ \\
\hline Acaena splendens & 1 & . & . & . & . & . & . & . & . & . & . & . & . & . & . & . & . & . & . & C & $\mathrm{P} / \mathrm{A}$ \\
\hline Maihueniopsis glomerata & II & 1 & . & . & . & . & . & . & . & . & . & . & . & . & . & . & . & . & . & $S$ & $P$ \\
\hline Rhodophiala mendocina & 1 & . & . & . & . & . & . & . & . & . & . & . & . & . & . & . & . & . & . & G & $M / P$ \\
\hline Maihuenia patagonica & 1 & . & 1 & . & . & . & . & . & . & . & . & . & . & . & . & . & . & . & . & S & $\mathrm{P}$ \\
\hline G2 & & & & & & & & & & & & & & & & & & & & & \\
\hline Larrea nitida & . & V & . & II & . & . & . & . & . & . & . & . & . & . & . & . & . & . & . & $\mathrm{N}$ & $M / P$ \\
\hline Lycium gilliesianum & . & 1 & . & . & . & . & . & . & . & . & . & . & . & . & . & . & . & . & . & $\mathrm{N}$ & $M / P$ \\
\hline Lecanophora ecristata & . & 1 & . & . & . & . & . & . & . & . & . & . & . & . & . & . & . & . & . & $\mathrm{T}$ & $\mathrm{M} / \mathrm{P}$ \\
\hline G3 & & & & & & & & & & & & & & & & & & & & & \\
\hline Colliguaja integerrima & . & II & V & II & . & . & . & . & . & . & . & . & . & . & . & . & . & . & . & $\mathrm{N}$ & $\mathrm{P}$ \\
\hline Schinus polygamus & 1 & V & IV & III & I & 1 & . & . & . & . & . & . & . & . & . & . & . & . & . & $\mathrm{N}$ & $\mathrm{P}$ \\
\hline Solanum triflorum & 1 & II & III & 1 & . & . & . & . & . & . & . & . & . & . & . & . & . & . & . & $\mathrm{T}$ & $\mathrm{P}$ \\
\hline Grindelia chiloensis & . & 1 & III & II & . & . & . & . & . & . & . & . & . & . & . & . & . & . & . & C & $M / P$ \\
\hline Astragalus pehuenches & . & . & II & 1 & 1 & . & . & . & . & . & . & . & . & . & . & . & . & . & . & C & $\mathrm{P}$ \\
\hline Senecio covasii & . & . & II & . & . & . & . & . & . & . & . & . & . & . & . & . & . & . & . & C & $\mathrm{P}$ \\
\hline Proustia cuneifolia & . & . & 1 & 1 & . & . & . & . & . & . & . & . & . & . & . & . & . & . & . & $\mathrm{N}$ & $\mathrm{M} / \mathrm{P}$ \\
\hline Solanum tweedianum & . & . & 1 & 1 & . & . & . & . & . & . & . & . & . & . & . & . & . & . & . & $\mathrm{T}$ & $\mathrm{M} / \mathrm{P}$ \\
\hline Sporobolus rigens & . & . & 1 & . & . & . & . & . & . & . & . & . & . & . & . & . & . & . & . & G & $\mathrm{M} / \mathrm{P}$ \\
\hline Berberis grevilleana & . & . & 1 & . & . & . & . & . & . & . & . & . & . & . & . & . & . & . & . & $\mathrm{N}$ & $\mathrm{P}$ \\
\hline Brachyclados lycioides & . & . & 1 & . & . & . & . & . & . & . & . & . & . & . & . & . & . & . & . & $\mathrm{N}$ & $\mathrm{P}$ \\
\hline Ephedra ochreata & . & . & 1 & . & . & . & . & . & . & . & . & . & . & . & . & . & . & . & . & $\mathrm{N}$ & $\mathrm{P}$ \\
\hline Nassauvia axillaris & . & . & 1 & . & . & . & . & . & . & . & . & . & . & . & . & . & . & . & . & $\mathrm{N}$ & P/A \\
\hline Monnina dictyocarpa & . & . & 1 & . & . & . & . & . & . & . & . & . & . & . & . & . & . & . & . & C & $\mathrm{M} / \mathrm{P}$ \\
\hline G4 & & & & & & & & & & & & & & & & & & & & & \\
\hline Fabiana imbricata & . & . & . & $\mathrm{V}$ & . & . & . & . & . & . & . & . & . & . & . & . & . & . & . & $\mathrm{N}$ & $\mathrm{P}$ \\
\hline Baccharis magellanica & . & . & . & II & . & . & . & . & . & . & . & . & . & . & . & . & . & & . & $\mathrm{N}$ & $\mathrm{P} / \mathrm{A}$ \\
\hline Acantholippia seriphioides & . & . & . & 1 & . & . & . & . & . & . & . & . & . & . & . & . & . & . & . & C & $\mathrm{M} / \mathrm{P}$ \\
\hline G5 & & & & & & & & & & & & & & & & & & & & & \\
\hline Adesmia volckmannii & . & . & . & . & $\mathrm{V}$ & 1 & I & 1 & . & . & . & . & . & . & . & . & . & . & . & $\mathrm{N}$ & $\mathrm{P}$ \\
\hline Schinus odonelli & . & . & 1 & . & II & 1 & . & . & 1 & . & . & . & . & . & . & . & . & . & . & $\mathrm{N}$ & $\mathrm{P}$ \\
\hline Jarava neaei & . & . & II & . & III & 1 & . & . & 1 & . & . & . & . & . & . & . & . & . & . & $\mathrm{H}$ & $\mathrm{P}$ \\
\hline Mulguraea ligustrina & . & . & . & . & II & 1 & . & . & 1 & . & . & . & . & . & . & . & . & . & . & $\mathrm{N}$ & $\mathrm{P}$ \\
\hline Loasa argentina & . & . & . & . & II & 1 & . & 1 & 1 & . & . & . & . & . & . & . & . & . & . & $\mathrm{T}$ & $\mathrm{P}$ \\
\hline Pilostyles berteroi & . & . & . & . & II & . & 1 & 1 & 1 & . & . & . & . & . & . & . & . & . & . & $\mathrm{P}$ & $\mathrm{P}$ \\
\hline Tropaeolum porifolium & . & . & . & . & II & 1 & . & . & . & . & . & . & . & . & . & . & . & . & . & $\mathrm{T}$ & $\mathrm{P}$ \\
\hline
\end{tabular}


Bol. Soc. Argent. Bot. 49 (2) 2014

\begin{tabular}{|c|c|c|c|c|c|c|c|c|c|c|c|c|c|c|c|c|c|c|c|c|c|}
\hline \multicolumn{22}{|c|}{ Especies características diagnósticas } \\
\hline Blumenbachia dissecta & . & . & . & . & II & . & . & . & . & . & . & . & . & . & . & 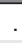 & . & . & . & $\mathrm{T}$ & $\mathrm{P}$ \\
\hline Poa ligularis & . & . & . & . & 1 & . & . & . & . & . & . & . & . & . & . & . & . & . & . & $\mathrm{H}$ & $\mathrm{P} / \mathrm{A}$ \\
\hline Jaborosa laciniata & . & . & . & . & 1 & . & . & . & . & . & . & . & . & . & . & . & . & . & . & $\mathrm{T}$ & $\mathrm{P} / \mathrm{A}$ \\
\hline Adesmia leptobotrys & . & . & . & . & 1 & . & . & . & . & . & . & . & . & . & . & . & . & . & . & $\mathrm{T}$ & $\mathrm{P} / \mathrm{A}$ \\
\hline Paronychia brasiliana & . & . & . & . & 1 & . & . & . & . & . & . & . & . & . & . & . & . & . & . & C & $\mathrm{M} / \mathrm{P}$ \\
\hline Convolvulus hermanniae & . & . & . & . & 1 & . & . & . & . & . & . & . & . & . & . & . & . & . & . & G & $\mathrm{P}$ \\
\hline \multicolumn{22}{|l|}{ G6 } \\
\hline $\begin{array}{l}\text { Chuquiraga } \\
\text { oppositifolia }\end{array}$ & 1 & . & . & . & 1 & V & 1 & II & II & II & . & . & . & . & . & . & . & . & . & $\mathrm{N}$ & $\mathrm{P} / \mathrm{A}$ \\
\hline Tropaeolum incisum & . & . & . & . & . & II & 1 & . & 1 & . & . & . & . & . & . & . & . & . & . & $\mathrm{T}$ & $\mathrm{P} / \mathrm{A}$ \\
\hline Pantacantha ameghinoi & . & . & . & . & . & 1 & . & . & 1 & . & . & . & . & . & . & . & . & . & . & $\mathrm{N}$ & $P$ \\
\hline Gutierrezia gilliesii & . & . & . & . & . & 1 & . & . & 1 & . & . & . & . & . & . & . & . & . & . & C & $\mathrm{M} / \mathrm{P}$ \\
\hline Oxalis compacta & . & . & . & . & . & 1 & . & 1 & . & . & . & . & . & . & . & . & . & . & . & $\mathrm{T}$ & $\mathrm{P} / \mathrm{A}$ \\
\hline Tetraglochin alatum & . & . & . & . & . & I & . & I & . & . & . & . & . & . & . & . & . & . & . & C & $\mathrm{P} / \mathrm{A}$ \\
\hline Festuca thermarum & . & . & . & . & & I & 1 & . & . & . & . & . & . & . & . & . & . & . & . & $\mathrm{H}$ & $\mathrm{P}$ \\
\hline Buddleja araucana & . & . & . & , & , & 1 & . & . & . & . & . & . & . & . & . & . & . & . & . & $\mathrm{N}$ & $\mathrm{P}$ \\
\hline Bowlesia tropaeolifolia & . & . & . & . & . & 1 & . & . & . & & . & & & & . & . & . & . & . & $\mathrm{T}$ & $M / P / A$ \\
\hline Melosperma andicola & . & . & . & . & . & I & . & . & . & . & . & . & . & . & . & . & . & . & . & $\mathrm{T}$ & $\mathrm{P} / \mathrm{A}$ \\
\hline Adesmia aff. aegiceras & . & . & . & . & . & 1 & . & . & . & . & . & . & . & . & . & . & . & . & . & $\mathrm{N}$ & $\mathrm{P} / \mathrm{A}$ \\
\hline Glandularia flava & . & . & . & . & . & 1 & . & . & . & . & . & . & . & . & . & . & . & . & . & C & $\mathrm{M} / \mathrm{P}$ \\
\hline Galium richardianum & . & . & . & . & . & 1 & . & . & . & . & . & . & . & . & . & . & . & . & . & C & $\mathrm{M} / \mathrm{P}$ \\
\hline \multicolumn{22}{|l|}{ G7 } \\
\hline Montiopsis cistiflora & . & . & . & . & . & . & III & . & . & I & . & . & . & 1 & . & . & . & . & . & C & A \\
\hline Adesmia corymbosa & 1 & . & . & . & . & I & II & I & . & I & . & . & . & . & . & . & . & . & . & C & $A$ \\
\hline Gayophyton micranthum & . & . & . & . & . & 1 & II & . & . & 1 & . & . & . & 1 & . & . & . & . & . & $\mathrm{T}$ & $\mathrm{P} / \mathrm{A}$ \\
\hline Boopis gracilis & . & . & . & . & . & 1 & ॥ & . & . & . & . & . & . & . & . & . & . & . & . & C & $P$ \\
\hline Tristagma nivale & . & . & . & . & . & . & 1 & . & . & . & . & I & . & . & . & . & . & . & . & G & A \\
\hline Adesmia capitellata & . & . & . & . & . & . & 1 & . & . & . & . & . & . & . & . & . & . & . & . & C & A \\
\hline $\begin{array}{l}\text { Hoffmannseggia } \\
\text { eremophila }\end{array}$ & . & . & . & . & . & . & 1 & . & . & . & . & . & . & . & . & . & . & . & . & G & $\mathrm{M} / \mathrm{P}$ \\
\hline Panicum urvilleanum & . & . & . & . & . & . & 1 & ${ }^{\circ}$ & . & . & . & . & . & & . & . & . & . & . & G & $M / P$ \\
\hline \multicolumn{22}{|l|}{ G8 } \\
\hline Adesmia pinifolia & . & . & . & . & . & II & . & . & V & . & . & . & . & . & . & . & . & . & . & $\mathrm{N}$ & $\mathrm{P} / \mathrm{A}$ \\
\hline $\begin{array}{l}\text { Oenothera } \\
\text { mendocinensis }\end{array}$ & . & . & . & . & . & . & . & . & I & . & . & . & . & . & . & . & . & . & . & $\mathrm{T}$ & A \\
\hline Schizanthus grahamii & . & . & . & . & . & . & . & . & 1 & . & . & . & . & . & . & . & . & . & . & $\mathrm{T}$ & $\mathrm{P} / \mathrm{A}$ \\
\hline \multicolumn{22}{|l|}{ G9 } \\
\hline Adesmia obovata & . & . & . & . & . & II & . & . & . & $\mathrm{V}$ & . & . & . & . & . & . & . & . & . & $\mathrm{N}$ & A \\
\hline Tweedia aucaensis & . & . & . & . & . & . & . & . & . & II & . & . & . & . & . & . & . & . & . & C & $\mathrm{M} / \mathrm{P}$ \\
\hline \multicolumn{22}{|l|}{ G10 } \\
\hline Nicotiana linearis & 1 & . & . & . & III & IV & II & I & 1 & II & . & . & . & . & . & . & 1 & . & . & $\mathrm{T}$ & $\mathrm{P}$ \\
\hline $\begin{array}{l}\text { Heliotropium } \\
\text { paronychioides }\end{array}$ & II & . & . & . & III & II & II & II & I & III & . & . & . & . & . & . & . & . & . & $\mathrm{T}$ & $\mathrm{P}$ \\
\hline Hordeum lechleri & . & . & . & . & III & . & 1 & II & 1 & . & . & . & . & . & . & . & . & . & I & $\mathrm{T}$ & $\mathrm{P}$ \\
\hline Acaena pinnatifida & . & . & . & . & 1 & I & I & I & 1 & I & . & . & . & . & . & . & I & . & . & C & $\mathrm{P} / \mathrm{A}$ \\
\hline Leymus erianthus & . & . & . & . & 1 & 1 & 1 & . & . & II & . & . & . & . & . & . & . & . & I & $\mathrm{H}$ & $M / P$ \\
\hline Calycera spinulosa & . & . & . & . & 1 & I & I & I & . & I & . & . & . & . & . & . & . & . & . & $\mathrm{T}$ & A \\
\hline Phacelia cumingii & . & . & . & . & 1 & 1 & 1 & 1 & 1 & . & . & . & . & . & . & . & . & . & . & $\mathrm{T}$ & A \\
\hline Polygala aff. stenophylla & . & . & . & . & 1 & 1 & II & I & . & . & . & . & . & . & . & . & . & . & . & C & $\mathrm{M} / \mathrm{P}$ \\
\hline Malesherbia lirana & . & . & . & . & 1 & 1 & . & . & I & . & . & . & . & . & . & . & . & . & . & G & $\mathrm{P} / \mathrm{A}$ \\
\hline Senna arnottiana & . & . & . & . & 1 & I & I & . & . & . & . & . & . & . & . & . & . & . & . & $\mathrm{C}$ & $P$ \\
\hline Doniophyton anomalum & . & . & . & . & 1 & 1 & . & 1 & . & . & . & . & . & . & . & . & . & . & . & $\mathrm{T}$ & $P$ \\
\hline Gilia crassifolia & . & . & . & . & . & 1 & I & I & I & . & . & . & . & . & . & . & . & . & . & $\mathrm{T}$ & $\mathrm{P} / \mathrm{A}$ \\
\hline Montiopsis gilliesi & . & . & . & . & . & 1 & 1 & I & . & 1 & . & I & . & . & . & . & . & . & . & C & $\mathrm{P} / \mathrm{A}$ \\
\hline Arjona patagonica & . & . & . & . & . & 1 & I & 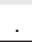 & . & I & . & . & . & . & . & . & . & . & . & G & $\mathrm{P} / \mathrm{A}$ \\
\hline
\end{tabular}


E. Méndez - Vegetación de los altos Andes Centrales

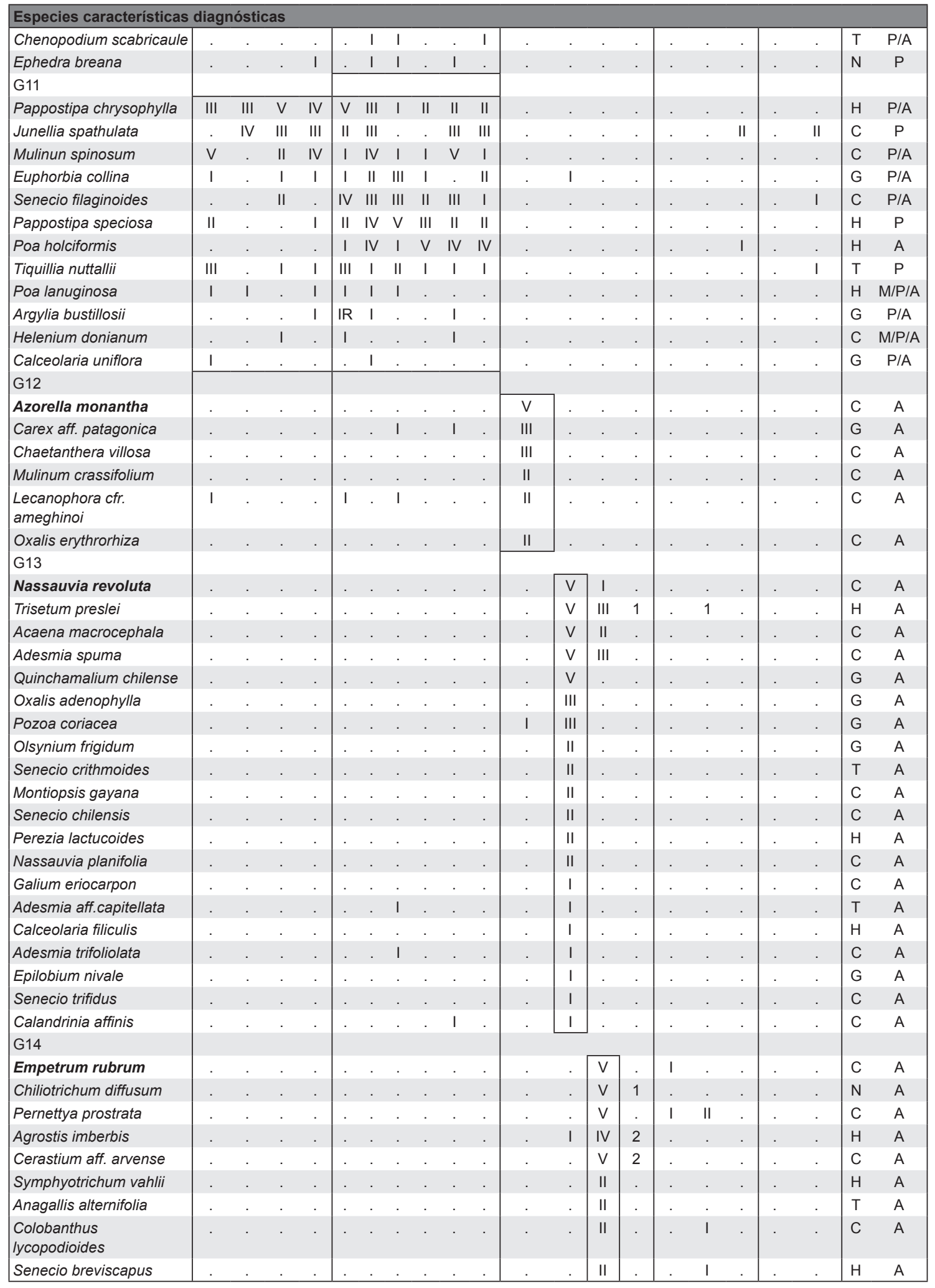


Bol. Soc. Argent. Bot. 49 (2) 2014

\begin{tabular}{|c|c|c|c|c|c|c|c|c|c|c|c|c|c|c|c|c|c|c|c|c|c|}
\hline \multicolumn{22}{|c|}{ Especies características diagnósticas } \\
\hline Erigeron patagonicus & . & . & . & . & . & . & . & . & . & . & . & 1 & II & . & . & . & . & . & . & $\mathrm{H}$ & A \\
\hline Senecio clarioneifolius & . & . & . & . & . & . & . & . & . & . & . & . & 1 & . & . & . & . & . & . & C & A \\
\hline Arenaria serpens & . & . & . & . & . & . & . & . & . & . & . & . & 1 & . & . & . & I & . & . & $\mathrm{T}$ & $\mathrm{P} / \mathrm{A}$ \\
\hline Cardamine aff. glacialis & . & . & . & . & & . & . & . & . & . & . & . & 1 & . & . & . & . & . & . & G & A \\
\hline Acaena magellanica & . & . & . & . & . & . & . & . & . & . & . & . & I & . & . & . & 1 & . & . & C & A \\
\hline Zameioscirpus sp. & . & . & . & . & . & . & . & . & . & . & . & I & 1 & . & . & 1 & . & . & . & G & A \\
\hline Carex vallis-pulchrae & . & . & . & . & . & . & . & . & . & . & . & . & I & . & . & 1 & . & . & . & G & A \\
\hline Poa planifolia & . & . & . & . & . & . & . & . & . & . & . & . & 1 & . & . & . & . & . & . & $\mathrm{H}$ & A \\
\hline Benthamiella patagonica & . & . & . & . & . & . & . & . & . & . & . & . & 1 & . & . & . & . & . & . & C & $\mathrm{P} / \mathrm{A}$ \\
\hline Oreopolus glacialis & . & . & . & . & . & . & . & . & . & . & . & . & 1 & . & . & . & . & . & . & C & A \\
\hline \multicolumn{22}{|l|}{ G15 } \\
\hline Ochetophila nana & . & . & . & . & . & . & . & . & . & . & . & . & II & 4 & & 1 & . & . & . & C & A \\
\hline Montiopsis aff. gayana & . & . & . & . & . & . & . & . & . & . & . & II & II & 2 & . & I & . & . & . & C & $A$ \\
\hline $\begin{array}{l}\text { Weberbauera } \\
\text { colchaguensis }\end{array}$ & . & . & . & . & . & . & . & . & . & . & . & . & . & 1 & . & . & . & . & . & $\mathrm{T}$ & A \\
\hline Calandrinia caespitosa & . & . & . & . & . & . & . & . & . & . & . & . & . & 1 & . & . & . & . & . & $\mathrm{T}$ & A \\
\hline Gamocarpha gilliesii & . & . & . & . & . & . & . & . & . & . & . & . & . & 1 & . & . & . & . & . & C & A \\
\hline Senecio grandjotii & . & . & . & . & . & . & . & . & . & . & . & . & . & $\mathrm{I}$ & . & . & . & & & C & A \\
\hline \multicolumn{22}{|l|}{ G16 } \\
\hline $\begin{array}{l}\text { Bromus setifolius } \\
\text { var.pictus }\end{array}$ & . & . & r & . & . & . & . & . & . & . & . & & 1 & 1 & . & . & . & . & . & $\mathrm{H}$ & A \\
\hline Euphrasia antarctica & . & . & . & . & . & . & . & . & . & . & . & . & 1 & 1 & . & . & . & . & . & C & A \\
\hline \multicolumn{22}{|l|}{ G17 } \\
\hline $\begin{array}{l}\text { Ranunculus } \\
\text { peduncularis }\end{array}$ & . & . & . & . & . & . & . & . & . & . & . & 1 & II & . & V & II & . & . & . & G & $\mathrm{P} / \mathrm{A}$ \\
\hline $\begin{array}{l}\text { Geranium aff. } \\
\text { sessiliflorum }\end{array}$ & . & . & . & . & . & . & . & . & . & . & . & . & . & . & V & 1 & . & . & . & G & A \\
\hline Olsynium aff. Junceum & . & . & . & . & . & . & . & . & . & . & . & . & . & . & IV & . & . & . & . & G & $A$ \\
\hline Plantago barbata & . & . & . & . & . & . & . & . & . & . & . & . & II & . & II & II & . & . & . & G & A \\
\hline Diplolepis nummulariifolia & . & . & . & . & . & . & . & . & . & . & . & . & . & . & II & . & . & . & . & C & A \\
\hline Cerastium aff. fontanum & . & . & . & . & . & . & . & . & . & . & . & . & . & . & II & 1 & . & . & . & C & $A$ \\
\hline Oxalis sp. & . & . & . & . & . & . & . & . & . & . & . & . & . & . & 1 & . & . & . & . & C & A \\
\hline Daucus pusillus & . & . & . & . & . & . & . & . & . & . & . & . & . & . & I & . & . & . & . & $\mathrm{T}$ & A \\
\hline Caltha sagittata & . & . & . & . & . & . & . & . & . & . & . & . & . & . & 1 & . & . & . & . & G & A \\
\hline \multicolumn{22}{|l|}{ G18 } \\
\hline Patosia clandestina & . & . & . & . & . & . & . & . & . & . & . & . & . & . & I & $\mathrm{V}$ & . & . & . & C & $A$ \\
\hline Festuca magellanica & . & . & . & . & . & . & . & . & . & . & . & . & I & 1 & . & III & . & . & . & $\mathrm{H}$ & $A$ \\
\hline Gentianella magellanica & . & . & . & . & . & . & . & . & . & . & . & . & II & . & . & III & . & . & . & G & A \\
\hline Phleum alpinum & . & . & . & . & . & . & . & . & . & . & . & . & 1 & . & . & III & . & . & . & $\mathrm{H}$ & $A$ \\
\hline Deschampsia caespitosa & . & . & . & . & . & . & . & . & . & . & . & . & 1 & 1 & . & III & 1 & . & . & $\mathrm{H}$ & $A$ \\
\hline Poa pratensis & . & . & . & . & . & . & . & . & . & . & . & . & . & . & . & II & . & 1 & . & $\mathrm{H}$ & $x$ \\
\hline Valeriana carnosa & . & . & . & . & . & . & . & . & . & . & . & . & . & . & . & 1 & . & . & . & G & $A$ \\
\hline Gentiana prostrata & . & . & . & . & . & . & . & . & . & . & . & . & . & . & . & 1 & . & . & . & G & A \\
\hline Koeleria mendocinensis & . & . & . & . & . & . & . & . & . & . & . & . & . & . & . & 1 & . & . & . & $\mathrm{H}$ & $A$ \\
\hline Colobanthus quitensis & . & . & . & . & . & . & . & . & . & . & . & . & . & . & . & 1 & . & . & . & C & A \\
\hline Anemone multifida & . & . & . & . & $\cdot$ & . & . & . & . & . & . & . & . & . & . & 1 & . & . & . & $\mathrm{H}$ & A \\
\hline Lobelia oligophylla & . & . & . & . & . & . & . & . & . & . & . & . & . & . & . & 1 & . & . & . & G & A \\
\hline \multicolumn{22}{|l|}{ G19 } \\
\hline Festuca kurtziana & . & . & . & . & . & . & . & . & . & . & . & . & . & . & . & 1 & V & . & . & $\mathrm{H}$ & A \\
\hline Carex patagonica & . & . & . & . & & . & . & . & . & . & . & . & . & . & . & . & II & . & . & G & $\mathrm{P} / \mathrm{A}$ \\
\hline Vicia pampicola & . & . & . & . & . & . & . & . & . & . & . & . & . & . & . & 1 & I & . & . & G & A \\
\hline Gamochaeta nivalis & . & . & . & . & . & . & . & . & . & . & . & . & . & . & . & 1 & 1 & . & . & $\mathrm{T}$ & A \\
\hline Deschampsia patula & . & . & . & . & . & . & . & . & . & . & . & . & . & . & . & 1 & 1 & . & . & $\mathrm{H}$ & A \\
\hline Rumex acetosella & . & . & . & . & . & . & . & . & . & & . & . & . & . & . & . & 1 & . & . & G & $x$ \\
\hline Azorella trifurcata & . & . & . & . & . & . & . & . & . & . & . & . & . & . & . & . & 1 & . & . & $\mathrm{C}$ & A \\
\hline
\end{tabular}


E. Méndez - Vegetación de los altos Andes Centrales

\begin{tabular}{|c|c|c|c|c|c|c|c|c|c|c|c|c|c|c|c|c|c|c|c|c|c|c|}
\hline \multicolumn{23}{|c|}{ Especies características diagnósticas } \\
\hline \multicolumn{23}{|c|}{\begin{tabular}{l|l}
$\mathrm{G} 20$ &
\end{tabular}} \\
\hline Olsynium junceum & & . & & & & & I & 1 & . & 1 & & I & $\mathrm{V}$ & III & 3 & IV & & & & & G & P/A \\
\hline Poa aff. holciformis & & & & & & & . & . & . & & & . & $\mathrm{v}$ & II & 4 & & & . & & & $\mathrm{H}$ & P/A \\
\hline Luzula racemosa & . & . & & & & & . & . & . & . & & . & IV & $\mathrm{v}$ & 2 & II & II & . & & . & $\mathrm{H}$ & A \\
\hline Perezia megalantha & . & . & & & & . & & & & . & . & . & 1 & $\mathrm{v}$ & 2 & . & . & & . & . & c & A \\
\hline Geranium sessiliflorum & . & . & & & & & IR & . & . & . & & & III & $\mathrm{V}$ & 1 & II & 1 & . & & . & C & A \\
\hline Silene echegarayi & . & . & & & & . & & & & & . & . & I & $\mathrm{V}$ & 1 & . & I & & & . & C & A \\
\hline Rytidosperma virescens & & . & & & & & . & . & . & 1 & & & ॥ & III & 1 & & . & 1 & & & $\mathrm{H}$ & $\mathrm{P} / \mathrm{A}$ \\
\hline Draba magellanica & & . & & & & & . & & . & . & & & 1 & 1 & 1 & 1 & . & & & & C & A \\
\hline Acaena aff. pinnatifida & . & . & & & & . & . & . & . & . & . & . & 1 & . & 1 & . & . & . & & . & C & A \\
\hline Senecio tricephalus & & . & & & & & . & & . & . & . & & 1 & 1 & . & & & & & & C & A \\
\hline Polygala williamsii & & & & & & & . & . & . & . & . & 1 & I & & . & & . & . & & . & C & A \\
\hline Leucheria achillaeifolia & & & & & & & & . & & . & & & I & & . & 1 & & . & & & $\mathrm{H}$ & A \\
\hline Armeria maritima & & & & & & & . & . & . & . & . & . & I & I & 1 & & & . & & . & C & A \\
\hline Acaena poeppigiana & & & & & & & & . & & . & . & . & . & 1 & . & 1 & . & . & & & C & A \\
\hline \multicolumn{23}{|l|}{ G21 } \\
\hline $\begin{array}{l}\text { Eleocharis } \\
\text { pseudoalbibracteata }\end{array}$ & . & . & & & & & . & . & . & & & . & & . & & & . & . & $\mathrm{v}$ & . & G & A \\
\hline Polypogon monspelienss & & . & & & & & . & . & . & . & . & . & . & . & . & & . & . & $\mathrm{v}$ & . & $\mathrm{T}$ & $x$ \\
\hline Trifolium repens & & . & & & & & . & . & . & & & . & & . & & & I & I & IV & . & C & $\mathrm{x}$ \\
\hline Phylloscirpus acaulis & . & . & & & & . & . & . & . & . & . & . & . & . & . & . & . & . & ॥ & . & G & P/A \\
\hline $\begin{array}{l}\text { Veronica anagallis- } \\
\text { aquatica }\end{array}$ & . & . & & & & & & . & . & . & & & . & . & $\cdot$ & r. & . & . & $\|$ & & G & $\mathrm{P} / \mathrm{A}$ \\
\hline \multicolumn{23}{|l|}{ G22 } \\
\hline Cortaderia rudiuscula & . & . & & & & . & & & & . & & . & . & . & . & . & . & & . & $\mathrm{V}$ & $\mathrm{N}$ & $M / P$ \\
\hline Lathyrus latifolius & . & . & & & & . & . & . & . & . & & . & . & . & . & . & . & . & & I & $\mathrm{T}$ & P/A \\
\hline Baccharis salicifolia & . & & & & & . & . & & . & . & . & . & . & . & . & . & & . & . & 1 & $\mathrm{~N}$ & $M / P / A$ \\
\hline $\begin{array}{l}\text { Bromus catharticus } \\
\text { var rupestris }\end{array}$ & & & & & & & . & . & . & . & & & . & . & . & & . & . & & 1 & $\mathrm{H}$ & $M / P / A$ \\
\hline Muhlenbergia asperifolia & & & & & & & . & & . & . & & & . & & . & & . & & & 1 & G & $M / P / A$ \\
\hline \multicolumn{23}{|l|}{ G23 } \\
\hline Juncus balticus & . & & & & & & 1 & & II & . & II & . & . & . & . & IV & II & V & $v$ & V & G & $M / P / A$ \\
\hline Carex gayana & & & & & & & . & . & . & . & . & & . & . & 1 & 1 & III & III & $\mathrm{v}$ & . & G & M/P/A \\
\hline \multicolumn{23}{|l|}{ Especies acompañantes } \\
\hline \multicolumn{23}{|l|}{24} \\
\hline Senecio subumbellatus & & & I & I & & $\mathrm{v}$ & V & IV & IV & IV & IV & ॥ & . & ॥ & 1 & & . & & I & ॥ & $\mathrm{N}$ & P/A \\
\hline Hordeum comosum & I & & & & & I & III & 1 & 1 & II & IV & ॥ & III & I & 2 & & I & II & ॥ & & $\mathrm{H}$ & P/A \\
\hline $\begin{array}{l}\text { Bromus setifolius } \\
\text { var. setifolius }\end{array}$ & & & & & & IIII & III & 1 & 1 & II & III & ॥ & 1 & . & . & & 1 & 1 & & & $\mathrm{H}$ & P/A \\
\hline Berberis empetrifolia & & & & & & & 1 & 1 & & I & ॥ & 1 & I & I & . & & I & . & & . & C & P/A \\
\hline Poa hиеси & & & & & & II & . & III & 1 & & I & II & & . & & & . & 1 & & III & $\mathrm{H}$ & P/A \\
\hline Calceolaria biflora & & & & & & & 1 & . & & 1 & . & $\theta$ & . & & 2 & I & I & I & & . & G & P/A \\
\hline Hypochaeris patagonica & & & & & & & 1 & 1 & I & I & 1 & 1 & 1 & 1 & & . & I & 1 & I & 1 & $\mathrm{H}$ & P/A \\
\hline Poa resinulosa & . & & & & & & 1 & . & . & 1 & . & . & . & . & . & . & . & . & . & . & $\mathrm{H}$ & $M / P / A$ \\
\hline Deschampsia flexuosa & & & & & & & . & . & & & & . & & & 1 & & I & I & & . & $\mathrm{H}$ & $\mathrm{P} / \mathrm{A}$ \\
\hline Moschopsis monocephala & & & & & & & . & 1 & & . & . & 1 & 1 & . & 1 & & . & . & & . & C & A \\
\hline Sisymbrium altissinum & & & & & & I & & 1 & . & 1 & & & & & & & . & I & & 1 & $\mathrm{~T}$ & $\mathrm{P} / \mathrm{A}$ \\
\hline Phacelia secunda & . & & & & & . & . & 1 & 1 & . & 1 & & 1 & . & 1 & 1 & . & . & & & C & $\mathrm{P} / \mathrm{A}$ \\
\hline \multicolumn{23}{|l|}{ Otros acompañantes } \\
\hline Centaurea solstitialis & & & & & & & . & . & . & . & & & . & . & & & . & . & & & $\mathrm{T}$ & $\mathrm{x}$ \\
\hline Bromus berteroanus & . & & & & & II & . & & & . & & & . & . & . & & . & & & & $\mathrm{T}$ & $\mathrm{x}$ \\
\hline Salsola kali & & & & & & . & . & . & . & 1 & & & . & . & . & & . & . & & & $\mathrm{T}$ & $\mathrm{x}$ \\
\hline Glicyrrhiza astragalina & & & & & & & . & . & & 1 & & & & & & & & . & & & G & $\mathrm{x}$ \\
\hline Cotula coronopifolia & & & & & & & & . & . & & & & & & & & & . & I & & T & $\mathrm{x}$ \\
\hline Sonchus oleraceus & . & & & & & . & . & . & & 1 & & & & 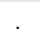 & & & & . & I & & $\mathrm{T}$ & $\mathrm{x}$ \\
\hline
\end{tabular}




\begin{tabular}{|c|c|c|c|c|c|c|c|c|c|c|c|c|c|c|c|c|c|c|c|c|c|}
\hline \multicolumn{22}{|c|}{ Especies características diagnósticas } \\
\hline Plantago lanceolata & . & . & . & . & . & . & . & . & . & . & . & . & . & . & . & . & . & 1 & . & $\mathrm{H}$ & $\mathrm{x}$ \\
\hline Anthemis cotula & . & . & . & . & . & . & . & . & . & . & . & . & . & . & . & . & . & 1 & . & $\mathrm{T}$ & $x$ \\
\hline Lactuca serriola & . & II & I & 1 & II & I & . & I & 1 & I & . & . & . & . & . & . & . & . & . & $\mathrm{T}$ & $x$ \\
\hline Cirsium vulgare & . & . & I & I & & I & . & . & 1 & . & . & . & . & . & . & I & I & . & I & $\mathrm{T}$ & $x$ \\
\hline Xanthium spinosum & . & . & II & I & 1 & I & . & . & . & . & . & . & & . & . & . & . & 1 & . & $\mathrm{T}$ & $\mathrm{x}$ \\
\hline Taraxacum officinale & . & . & . & I & . & . & । & I & 1 & . & . & . & 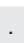 & . & . & . & 1 & I & . & $\mathrm{H}$ & $x$ \\
\hline Onopordon acanthium & . & . & . & . & I & 1 & . & . & . & I & . & . & . & . & . & . & . & . & . & $\mathrm{T}$ & $\mathrm{x}$ \\
\hline Erodium cicutarium & . & . & . & . & . & I & . & II & 1 & . & . & . & & . & . & . & . & . & . & $\mathrm{T}$ & $x$ \\
\hline Dysphania ambrosioides & . & I & . & 1 & . & . & . & . & . & . & . & . & . & . & . & . & . & 1 & . & $\mathrm{T}$ & $x$ \\
\hline Gnaphalium sp. & . & . & . & 1 & . & . & . & . & . & . & . & . & . & . & . & . & I & I & . & C & $\mathrm{x}$ \\
\hline Cerastium arvense & . & . & . & . & . & . & . & . & . & . & . & . & . & 2 & I & I & . & . & . & C & $x$ \\
\hline
\end{tabular}

Pisos vegetales de 1: comunidades $1,2,3$, y 4; 2: comunidades $5,6,7,8,9$, y 10; 3: comunidades $11,12,13$ y 14; 4: de las vegas de altura de 15,16 y $17 ; 5$ de las vegas de baja altura, de 18 y 19. Comunidades vegetales de 1: Neosparton ephedroides, 2: Larrea nitida, 3: Colliguaya integerrima, 4: Fabiana imbricata, 5: Adesmia volckmannii, 6: Chuquiraga oppositifolia, 7: Pappostipa speciosa, 8: Poa holciformis, 9: Adesmia pinifolia, 10: Adesmia obovata, 11: Azorella monantha, 12: Nassauvia revoluta, 13: Empetrum rubrum, 14: Ochetophila nana, 15: Ranunculus peduncularis, 16: Patosia clandestina, 17: Festuca kurtziana,18: Juncus balticus, 19: Cortaderia rudiuscula. A: formas de vida: T: Terófita, C: Caméfita, G: Geófita, H: Hemicriptófita, N: Nanofanerófita, S: Suculenta, E: Epífita, P: Parásita. B: Provincia corológica: A: Altoandino o andino, M: Monte, P. Patagonica, X: Exóticas.

Tabla 2. Distribución de las formas de vida en las comunidades vegetales de Bardas Blancas-Paso Pehuenche.

\begin{tabular}{|c|c|c|c|c|c|c|c|c|}
\hline$A / B$ & $\mathrm{~T}$ & $\mathrm{H}$ & C & G & $\mathbf{N}$ & $\mathrm{S}$ & $P$ & Total \\
\hline 1 & 7 & 4 & 3 & 3 & 3 & 2 & . & 22 \\
\hline 2 & 5 & 2 & 2 & . & 4 & 1 & . & 14 \\
\hline 3 & 7 & 2 & 8 & 2 & 9 & 1 & . & 29 \\
\hline 4 & 8 & 5 & 6 & 3 & 7 & . & . & 29 \\
\hline 5 & 16 & 12 & 9 & 4 & 6 & . & 1 & 48 \\
\hline 6 & 18 & 13 & 14 & $\underline{9}$ & $\underline{12}$ & . & . & 66 \\
\hline 7 & 12 & 11 & 15 & 7 & 4 & . & 1 & 50 \\
\hline 8 & 12 & 9 & 7 & 2 & 3 & . & 1 & 34 \\
\hline 9 & 15 & 11 & 8 & 6 & 7 & . & 1 & 48 \\
\hline 10 & 9 & 8 & 9 & 3 & 3 & . & . & 32 \\
\hline 11 & . & 4 & 8 & 3 & 1 & . & . & 16 \\
\hline 12 & 2 & 12 & 23 & $\underline{10}$ & . & . & . & 47 \\
\hline 13 & 2 & 15 & 22 & 7 & 2 & . & . & 48 \\
\hline 14 & 3 & 10 & 16 & 3 & 2 & . & . & 34 \\
\hline 15 & 1 & 2 & 11 & 9 & . & . & . & 23 \\
\hline 16 & 2 & 15 & 12 & 13 & . & . & . & 42 \\
\hline 17 & 4 & 12 & 6 & 6 & . & . & . & 28 \\
\hline 18 & 6 & 5 & 2 & 5 & 1 & . & . & 19 \\
\hline 19 & 4 & 5 & 2 & 2 & 3 & . & . & 16 \\
\hline
\end{tabular}

A: Formas de vida: T: Terófita, H: Hemicriptófita. C: Caméfita, G: Geófita, N: Nanofa-nerófita, S: Suculenta, P: Parásita. B: Comunidades vegetales de: 1: Neosparton ephedroides, 2: Larrea nitida, 3: Colliguaja integerrima; 4: Fabiana imbricata; 5: Adesmia volckmannii, 6: Chuquiraga oppositifolia, 7: Pappostipa speciosa; 8: Poa holciformis; 9: Adesmia pinifolia; 10: Adesmia obovata, 11: Azorella monantha; 12: Nassauvia revoluta, 13: Empetrum rubrum, 14: Ochetophila nana, 15: Ranunculus peduncularis; 16: Patosia clandestina, 17: Festuca kurtziana, 18: Juncus balticus; 19: Cortaderia rudiuscula. 


\begin{tabular}{|c|c|c|c|}
\hline \multicolumn{4}{|c|}{$\begin{array}{c}\text { Tabla 3. Valores promedios de altitudes } \\
\text { y temperaturas medias anuales de las } \\
\text { comunidades vegetales de Bardas Blancas-Paso } \\
\text { Pehuenche. }\end{array}$} \\
\hline $\mathbf{N}^{0}$ de orden & $\underset{\mathrm{N}^{\circ}}{\text { Comunidad }}$ & $\begin{array}{l}\text { Altitud m } \\
(1=10 \mathrm{~m})\end{array}$ & $\begin{array}{l}\text { Temperatura } \\
{ }^{\circ} \mathrm{C}{ }^{*} \\
\text { media anual }\end{array}$ \\
\hline 1 & 2 & 145 & 11.2 \\
\hline 2 & 4 & 147 & 11.1 \\
\hline 3 & 3 & 148 & 11.0 \\
\hline 4 & 1 & 150 & 10.9 \\
\hline 5 & 5 & 161 & 9.7 \\
\hline 6 & 18 & 172 & 9.5 \\
\hline 7 & 19 & 178 & 9.4 \\
\hline 8 & 8 & 187 & 9.2 \\
\hline 9 & 9 & 190 & 9.0 \\
\hline 10 & 6 & 195 & 8.8 \\
\hline 11 & 7 & 199 & 8.1 \\
\hline 12 & 17 & 215 & 6.9 \\
\hline 13 & 10 & 225 & 6.3 \\
\hline 14 & 16 & 247 & 5.1 \\
\hline 15 & 12 & 252 & 4.5 \\
\hline 16 & 13 & 254 & 4.3 \\
\hline 17 & 14 & 256 & 4.2 \\
\hline 18 & 15 & 258 & 4.0 \\
\hline 19 & 11 & 276 & 3.3 \\
\hline
\end{tabular}

Valores de altitudes promedios de cada comunidad obtenidos de los relevamientos de las tablas parciales. Valores de temperaturas promedios de Defina et al (1964) y estimados según $-0,6^{\circ} \mathrm{C} / 100 \mathrm{~m}$ de altitud (Corte , 1984).

Tabla 4. Distribución de los elementos
corológicos en las comunidades vegetales de
Bardas Blancas-Paso Pehuenche.
\begin{tabular}{|cccccccc|c|}
\hline Comunidad & P & M/P & PIA & X & M/PIA & A & Total \\
\hline 1 & 9 & 3 & 7 &. & 1 & 2 & 22 \\
2 & 5 & 6 &. & 2 & 1 &. & 14 \\
3 & 13 & 6 & 6 & 3 & 1 &. & 29 \\
4 & 9 & 5 & 5 & 8 & 2 &. & 29 \\
5 & 19 & 3 & 16 & 4 & 2 & 4 & 48 \\
6 & 20 & 5 & 27 & 6 & 3 & 5 & 66 \\
7 & 11 & 4 & 22 & 1 & 1 & 11 & 50 \\
8 & 9 & 1 & 16 & 4 &. & 4 & 34 \\
9 & 13 & 1 & 20 & 7 & 2 & 5 & 48 \\
10 & 5 & 2 & 17 & 3 &. & 5 & 32 \\
11 &. &. & 8 &. &. & 35 & 16 \\
12 &. &. & 12 &. &. & 29 & 47 \\
13 &. &. & 10 &. &. & 48 & 48 \\
14 &. &. & 9 &. & 1 & 24 & 34 \\
15 &. &. & 4 & 2 & 1 & 16 & 23 \\
16 &. &. & 7 & 5 & 1 & 29 & 42 \\
\cline { 5 - 7 } 17 & 2 & & 11 & 5 & 1 & 9 & 28 \\
18 &. &. & 5 &. & 14 & 1 & 20 \\
19 & 3 & 2 & 6 & 1 & 4 &. & 16 \\
\hline
\end{tabular}

Elementoscorológicos: M: delMonte,P:Patagonica, A: Alto Andino, $\mathrm{X}$ : exóticas. Comunidades vegetales de 1: Neosparton ephedroides, 2: Larrea nitida, 3: Colliguaja integerrima, 4: Fabiana imbricata, 5 Adesmia volckmannii, 6: Chuquiraga oppositifolia, 7: Pappostipa speciosa, 8: Poa holciformis, 9: Adesmia pinifolia, 10: Adesmia obovata, 11: Azorella monantha, 12: Nassauvia revoluta, 13: Empetrum rubrum, 14: Ochetophila nana, 15: Ranunculus peduncularis, 16: Patosia clandestina, 17: Festuca kurtziana, 18: Juncus balticus, 19: Cortaderia rudiuscula.

Tabla 5. Distribución de los elementos corológicos en los pisos vegetales de Bardas Blancas-Paso Pehuenche.

\begin{tabular}{|c|c|c|c|c|c|c|c|}
\hline Piso Veg. & $\mathrm{P}$ & $\mathrm{M} / \mathrm{P}$ & P/A & X & M/P/A & A & Total \\
\hline 1 & 20 & 12 & 12 & 7 & 4 & 2 & 57 \\
\hline 2 & 24 & 9 & 36 & 10 & 4 & 15 & 98 \\
\hline 3 & . & . & 18 & 1 & 1 & 70 & 90 \\
\hline \multicolumn{8}{|c|}{ De las vegas } \\
\hline 4 & 1 & . & 15 & 7 & 2 & 41 & 66 \\
\hline 5 & 2 & 2 & 8 & 12 & 4 & & 28 \\
\hline
\end{tabular}

Elementos corológicos: M:del Monte, P: Patagonica, A: Andino; X: exóticas. Pisos vegetales de 1: de las comunidades de Neosparton ephedroides, Larrea nitida, Colliguaja integerrima y de Fabiana imbricata, 2 : de las comunidades de: Adesmia volckmannii, Chuquiraga oppositifolia, Pappostipa speciosa, Poa holciformis, Adesmia pinifolia y de Adesmia obovata; 3 de las comunidades de: Azorella monantha, Nassauvia recurvata, Empetrum rubrum, y Ochetophila nana, Piso de las vegas, 4: comunidades de altura de: Ranunculus peduncularis, Patosia clandestina, Festuca kurtziana, 5: de las comunidades vegetales de vegas de baja altura de: Juncus balticus y de Cortaderia rudiuscula. 


\section{Agradecimientos}

A dos revisores anónimos que evaluaron el trabajo y dieron valiosas sugerencias para mejorarlo, a Oscar R. Estévez por la asistencia técnica en los cálculos estadísticos, a Nélida Horak por la traducción del resumen al inglés y a Cecilia Scoones por la transcripción de los dibujos. A los puesteros de la zona que brindaron y facilitaron valiosa ayuda como guías durante las campañas de campo.

\section{Bibliografía}

AMBROSETTI, J. A., L. A. DEL VITTO \& F. A. ROIG. 1986. La vegetación del Paso de Uspallata, Provincia de Mendoza, Argentina.Veröff. Geobot. Inst. Eth. Stiftung Rubel, Zurich 91: 141-180.

BÖECHER, T. W., J. P. HJERTING \& K. RHAN. 1972. Botanical Studies in The Atuel Valley Area, Mendoza, Province Argentina. Dansk Botanisk Ark 22: 1 (1-125), 22: 2 (121-185), 22: 3 (191-358).

BORDONARO, O. L. 2012. Mundos extinguidos de Mendoza. Zeta Editores, Mendoza.

BRAUN-BLANQUET, J. 1979. Fitosociología. Bases para el estudio de las comunidades vegetales. $\mathrm{H}$. Blume, Madrid.

CABRERA, A. L. 1976. Regiones Fitogeográficas de la República Argentina. En: Kluger W. F. (ed.), Enciclopedia Argentina de Agricultura y Jardinería 2 (1): 1-85. $2^{\mathrm{a}}$ ed. ACME, Buenos Aires.

CAPITANELLI, R. 1972. Geomorfología y clima de la provincia de Mendoza. Las Ciencias Naturales en la Provincia de Mendoza. Bol. Soc. Argent. Bot. 13 (Supl.): 15-48.

CHIAPELLA, J. \& C. EZCURRA.1999. La flora del Parque Provincial Tromen, Provincia de Neuquén, Argentina. Multequina 8: 51-60.

CORTE, A. 1983a. Los conceptos geocriogénicoparageocriogénico y glacial-periglacial en los Andes Centrales de Argentina, latitud $30^{\circ}$. Anales IANIGLA 83: 48-61.

CORTE, A. 1983b. Geocriología. El Frio en la Tierra. Ediciones culturales de Mendoza, Ediunc, Mendoza.

DE FINA, A. L., F. GIANNETTO, A. E. RICHARD \& L. S. SABELLA. 1964. Difusión geográfica de los cultivos índices de la provincia de Mendoza y sus causas. INTA, Inst. de Suelos y Agrotecnia, Mendoza.

DE MARCO, G., F. A. ROIG \& C. WUILLOUD. 1993. Vegetación del Piedemonte andino en el centro oeste de Mendoza (68 $32-69^{\circ} 22$ de long W y $33^{\circ} 42-3440$ lat. S). Multequina 2: 201-242.
DESSANTI, R. M. 1973. Descripción geológica de la hojas 29b Bardas Blancas. Provincia de Mendoza Carta geológica-Económica de la República Argentina. Escala 1:200.000, 1-70. Buenos Aires.

DI RIENZO, J. A., F. CASANOVES, M. G. BALZARINI, L. GONZÁLEZ, M. TABLADA \& C. W. ROBLEDO. 2009. InfoStat Versión 2010. FCA, Universidad Nacional de Córdoba.

FERREYRA, M., S. CLAYTON \& C. EZCURRA.1998. La flora altoandina de los sectores este y oeste del Parque Nacional Nahuel Huapi, Argentina. Darwiniana 36: 65-79.

GONZÁlEZ DIAZ, E. F. \& L. E. FAUQUE. 1993. Geomorfología. XII Congreso Argentino de Exploración de Hidrocarburos, Geología y Recursos naturales de Mendoza. V. A. Ramos (ed.), Relatorio 1 (7): 217-234.

HAUMANN, L. 1918. La végétation des Hautes Cordillères de Mendoza. Anales Soc. Ci. Argent. 86: 121-188, 255-348.

LACOSTE, P. 1998. El sistema Pehuenche: Frontera, Sociedad y Caminos en los Andes Centrales Argentino Chileno (1658-1997). Ediciones culturales de Mendoza, Ediunc, Mendoza.

LEON, R. J. C., D. BRAN, M. COLLANTES, J. M. PARUELO y A. SORIANO. 1998. Grandes unidades de vegetación de la patagonia extra endina. Ecol. Austral 8: 125-144.

MARTINEZ CARRETERO, E. 2001. Vegetación de los Andes Centrales de la Argentina. El Valle de Uspallata, Mendoza. Bol. Soc. Argent. Bot. 34: 127-148.

MARTINEZ CARRETERO, E. \& E. MÉNDEZ. 1992. La vegetación de la vertiente oriental de la Cordillera Real, Mendoza-Argentina. Multequina 1: 99-106.

MARTÍNEZ CARRETERO, E. 2004. La provincia Fitogeográfica de la Payunia. Bol. Soc. Argent. Bot. 39: 195-226.

MÉNDEZ, E. \& C. WUILLOUD. Inédito. Carta de vegetación El Manzano 1:100.000, Mendoza.

MÉNDEZ, E. R., F. ROIG, \& E. MARTINEZ CARRETERO. 1988. Mapa florístico. Provincia de Mendoza. Diario Los Andes.

MÉNDEZ, E. 2004. La vegetación de los Altos Andes. I Pisos de vegetación del flanco oriental del Cordón del Plata, Mendoza Argentina. Bol. Soc. Argent. Bot. 39: 227- 253.

MÉNDEZ, E. 2007. La vegetación de los Altos Andes. II Las vegas del flanco oriental del Cordón del Plata, Mendoza, Argentina. Bol. Soc. Argent. Bot. 42: 273- 294.

MÉNDEZ, E. 2009. Biodiversidad de la Flora del flanco oriental del Cordón del Plata (Luján de Cuyo, Mendoza, Argentina). Bol. Soc. Argent. Bot. 44: 75-102.

MÉNDEZ, E. 2010. La vegetación saxícola y de taludes 


\section{E. Méndez - Vegetación de los altos Andes Centrales}

del flanco oriental del Cordón del Plata (Luján de Cuyo, Mendoza, Argentina). Bol. Soc. Argent. Bot. 45: 119-147.

MÉNDEZ, E. 2011. Crecimiento y recubrimiento de Azorella monantha Clos (Apiaceae) en los altos Andes Centrales de Mendoza, Argentina. Revista Fac. Ci. Agrar. UNCuyo 43: 219-229.

MÉNDEZ, E., E. MARTÍNEZ CARRETERO \& I. PERALTA. 2006. La vegetación del Parque Provincial Aconcagua (Altos Andes Centrales de Mendoza, Argentina). Bol. Soc. Argent. Bot. 41: 41-69.

MINETTI, J. H. \& A. E. CORTE. 1984. Zonificación latialtitudinal del clima en la zona andina y su relación con el límite inferior del hielo perenne (LIHP) y del límite inferior geocriogénico (LIG). Anales IANIGLA 6: 129-144.

MOVIA, C., G. H. OWER \& C. E. PEREZ. 1982. Estudio de la vegetación natural de la Provincia del Neuquén, Relevamiento 1y 2. Informe inédito de la Subsecretaría de Recursos Naturales, Neuquén.

MUELLER-DOMBOIS, D. \& H. ELLENBERG. 1974. Aims and methods of vegetation ecology. J. Wiley \& Sons, New York.

NORTE, F. 2000. Mapa climático de Mendoza. En: Abraham, E. M. \& F. M. Martínez (eds.), Argentina. Recursos y Problemas Ambientales de las Zonas Áridas. Primera parte. Provincias de Mendoza, San Juan y La Rioja. Tomo I: Caracterización Ambiental Argentina 1: 25-27GTZ, IDR (Univ. Granada) IADIZA, SDSYPA, Buenos Aires.

POLANSKI, J. 1963. Carta geológica-económica Volcán San José.1:200.000. Min. de Economía, Dirección General de Minería, Buenos Aires.

RAUNKIAER, C. 1934. Life forms and terrestrial plant geography. Clarendon Press, Oxford.

ROIG, F. A. 1960. Bosquejo fitogeográfico de la provincia de Mendoza. Offsett.

ROIG, F. A. 1972. Flora de Ñancuñán. Deserta 1: 1-289.

ROIG, F. A. 1976. Las comunidades vegetales del Piedemonte de la Precordillera de Mendoza. Ecosur 3: $1-45$.
ROIG, F. A. 1989. Ensayo de detección y control de la desertificación en el W de la ciudad de Mendoza desde el punto de vista de la vegetación. Conferencias, trabajos y resultados del Curso Latinoamericano Detección y Control de la Desertificación. UNEPIADIZA: 196-232.

ROIG, F. A. \& E. MARTINEZ CARRETERO. 1998. La vegetación puneña en la provincia de Mendoza, Argentina. Phytocoenología 28: 565-608.

RUIZ LEAL, A. R. 1961. Un oasis en el desierto. Bol. Est. Geogr. UNcuyo 8 (32): 97-108.

RUIZ LEAL, A. R. 1972. Los confines boreal y austral de las provincias Patagónica y Central respectivamente. Bol. Soc. Argent. Bot. 13: 88-118.

RUIZ LEAL, A. R. 1973. Flora popular mendocina. Deserta 3:1-296.

SORIANO, A. 1956. Los distritos florísticos de la Provincia Patagonica. Revista Invest. Agrop. 10: 323-347.

ZULOAGA, F. O., O. MORRONE \& M. J. BELGRANO (eds.). 2008a. Catálogo de las Plantas Vasculares del Cono Sur (Argentina, Sur de Brasil, Chile, Paraguay y Uruguay). Pteridophyta, Gymnospermae, Monocotyledoneae. Monogr. Syst. Bot. Missouri Bot. Gard. 107, vol. 1: 1-983.

ZULOAGA, F. O., O. MORRONE \& M. J. BELGRANO (eds.). 2008b. Catálogo de las Plantas Vasculares del Cono Sur (Argentina, Sur de Brasil, Chile, Paraguay y Uruguay). Dicotyledoneae: Acanthaceae-Fabaceae (Abarema-Schizolobium), Monogr. Syst. Bot. Missouri Bot. Gard. 107 vol. 2: 985-2286.

ZULOAGA, F. O., O. MORRONE \& M. J. BELGRANO (eds.). 2008c. Catálogo de las Plantas Vasculares del Cono Sur (Argentina, Sur de Brasil, Chile, Paraguay y Uruguay) Dicotyledoneae: Fabaceae (Senna-Zygia)- Zygophyllaceae, Monogr. Syst. Bot. Missouri Bot. Gard. 107 vol. 3: 2287-3348.

Recibido el 14 de agosto de 2013, aceptado el 28 de marzo de 2014. 
